\title{
Algebro-Geometric Solutions of the Generalized Virasoro Constraints
}

\author{
Francisco José PLAZA MARTÍN \\ Departamento de Matemáticas and IUFFYM, Universidad de Salamanca, \\ Plaza de la Merced 1-4, 37008 Salamanca, Spain \\ E-mail: fplaza@usal.es \\ URL: http://mat.usal.es/ fplaza/
}

Received December 20, 2014, in final form July 02, 2015; Published online July 07, 2015

http://dx.doi.org/10.3842/SIGMA.2015.052

\begin{abstract}
We will describe algebro-geometric solutions of the KdV hierarchy whose $\tau$ functions in addition satisfy a generalization of the Virasoro constraints (and, in particular, a generalization of the string equation). We show that these solutions are closely related to embeddings of the positive half of the Virasoro algebra into the Lie algebra of differential operators on the circle. Our results are tested against the case of Witten-Kontsevich $\tau$ function. As by-products, we exhibit certain links of our methods with double covers of the projective line equipped with a line bundle and with $\mathrm{Gl}(n)$-opers on the punctured disk.
\end{abstract}

Key words: string equation; Virasoro constraints; KP hierarchy; Gl( $n)$-opers; Sato Grassmannian; topological recursion

2010 Mathematics Subject Classification: 14H71; 14D24; 81R10; 81T40

\section{Introduction}

In recent decades, the relevant role in mathematics and in physics of the class of functions that simultaneously satisfy the KdV-hierarchy and the Virasoro constraints has been discovered (as a mere illustration, let us cite $[8,10,12,21,25])$. On the one hand, the KdV-hierarchy is currently quite well understood after the works of Sato and it has been successfully applied in topics such as Shiota's solution of the Schottky problem. On the other hand, representation theory of Virasoro algebras has been studied in depth; in particular its study has led to significant results in the theory of vertex operator algebras and in the geometric Langlands program. From the point of view of mathematical physics, these types of equations appear naturally in conformal field theory when studying the symmetries of objects.

One principal character of this story is played by a particular function in each theory. Among the variety of problems in which both families of equations, KdV and Virasoro, appear together, let us pick up the functions of two of them: the partition function of $2 \mathrm{D}$ gravity and the generating functions for intersection numbers. The fact is that these functions are $\tau$-functions for the KdV-hierarchy and solutions to the Virasoro constraints. Indeed, this is an excellent example to illustrate the deep consequences emerging from their study. Recall, for instance, the Witten conjecture, proved by Kontsevich, asserting that both $\tau$-functions do coincide or the implications in the Gromov-Witten theory and enumerative geometry thanks to the ELSV formula, which links Hurwitz numbers and Hodge integrals.

Within the context of matrix models, Eynard and Orantin $[12,13]$ have recently shown that starting from an arbitrary spectral curve one can construct many objects of the theory. In particular, the nonperturbative partition function is closely related to the theta function of the curve and that, again, it is a $\tau$-function for the KdV-hierarchy that solves the Virasoro constraints [11]. 
At this point we wondered about developing a general formalism for the study of the KdVVirasoro combination with emphasis on the role of the $\tau$-function. Here and henceforth, under Virasoro constraints for $\tau(t)$ we mean there exist a family of differential operators $\left\{\bar{L}_{k}\right\}_{k \geq-1}$, satisfying the relations of the Virasoro algebra, such that $\bar{L}_{k} \tau(t)=0$ for $k \geq-1$. Thus, the Virasoro constraints in 2D quantum gravity as in [8,21,25] correspond to a particular choice of $\left\{\bar{L}_{k}\right\}_{k \geq-1}$. The present paper aims to offer a first step in this program. The moral of the paper is that there are close and explicit relations among the following sets:

$$
\begin{gathered}
\left\{\begin{array}{c}
\tau(t) \in \mathbb{C}\left[\left[t_{1}, t_{2}, \ldots\right]\right] \\
\text { satisfying } \mathrm{KdV} \text { and } \\
\text { Virasoro constraints } \\
\mid C
\end{array}\right\} \underset{B}{\stackrel{A}{<---}}\left\{\begin{array}{c}
\text { subalgebras }<\left\{\tilde{L}_{n}\right\}_{n \geq-1}> \\
\text { of } \mathcal{D}_{\mathbb{C}((z)) / \mathbb{C}}^{1}(\mathbb{C}((z)), \mathbb{C}((z))) \\
\text { with }\left[\tilde{L}_{i}, \tilde{L}_{j}\right]=(i-j) \tilde{L}_{i+j}
\end{array}\right\} \\
\left\{\begin{array}{c}
2: 1 \text {-covers } X \rightarrow \mathbb{P}_{1} \text { and a } \\
\text { rank } 1 \text { torsion free sheaf on } X
\end{array}\right\} \quad\left\{\begin{array}{c}
\operatorname{Gl}(2) \text {-opers } \\
\text { on } \operatorname{Spec} \mathbb{C}\left(\left(z^{2}\right)\right)
\end{array}\right\}
\end{gathered}
$$

where we shall skip the details until later.

Let us discuss naively how the above arrows are constructed and postpone the rigor for the heart of the paper. Roughly, the map $A$ from functions to subalgebras is a forgetful map. More precisely, observe that saying that $\tau(t)$ is a $\tau$-function for the $\mathrm{KdV}$ that solves the Virasoro constraints means that $\tau(t) \in \mathbb{C}\left[\left[t_{1}, t_{2}, \ldots\right]\right]$ satisfies the following equations:

(i) KP-hierarchy,

(ii) $\partial_{t_{2 i}} \tau(t)=0$ (KdV hierarchy, provided that $\mathrm{KP}$ is fulfilled),

(iii) $\bar{L}_{k} \tau(t)=0$, for $k \geq-1$ (Virasoro constraints), for certain differential operators $\left\{\bar{L}_{k}\right\}_{k \geq-1}$ with $\left[\bar{L}_{i}, \bar{L}_{j}\right]=(i-j) \bar{L}_{i+j}$.

Recall that, in the case of $2 \mathrm{D}$ quantum gravity, the equation $\bar{L}_{-1} \tau(t)=0$ is the so called string equation. The following convention will used; $L_{i}$ will denote a set of generators of the Witt algebra, as an abstract Lie algebra, such that $\left[L_{i}, L_{j}\right]=(i-j) L_{i+j} ; \tilde{L}_{i}$ will be regarded as fields acting on $\mathbb{C}((z))$ with the same Lie bracket; and, finally, $\bar{L}_{i}$ will be fields acting on the Fock space $\mathbb{C}\left[\left[t_{1}, t_{2}, \ldots\right]\right]$ with the same Lie bracket.

Now, the bosonization isomorphism provides us with a method to transport operators, $\bar{L}_{k}$, acting on $\mathbb{C}\left[\left[t_{1}, t_{2}, \ldots\right]\right]$ to first-order differential operators on $\mathbb{C}((z))$, which we denote by $\tilde{L}_{k}$. Thus, $\left\{\tilde{L}_{k}\right\}_{k \geq-1}$ is the desired subalgebra of the map $A$.

The difficult part is $B$, from subalgebras to functions, since we need to construct a function. For this goal the Sato theory of soliton equations will be relied on heavily. Recall that the Sato theory claims that $\tau$-functions for the KP hierarchy correspond bijectively to certain subspaces of $\mathbb{C}((z))$ and that, moreover, these subspaces are precisely the points of an infinite-dimensional Grassmann manifold, $\operatorname{Gr}(\mathbb{C}((z)))$. Hence, instead of looking for $\tau$-functions for the KP we may look for subspaces $U \subseteq \mathbb{C}((z))$ lying in the Grassmannian of $\mathbb{C}((z))$ and, at the end of the day, the desired $\tau$-function will appear as the $\tau$-function associated with the point $U, \tau_{U}(t)$. Thus, our strategy consists of restating the problem in terms of subspaces of $\mathbb{C}((z))$ with the help of Sato theory. Provided that the KP hierarchy is fulfilled, the KdV hierarchy for $\tau(t)$ can be explicitly written in terms of subspaces; indeed, for a subspace $U$ it is equivalent to the condition $z^{-2} U \subseteq U$.

So far, the problem of constructing the map $B$ can be restated as follows: given a Lie subalgebra $\left\langle\left\{\tilde{L}_{k}\right\}_{k \geq-1}\right\rangle$ of $\mathcal{D}_{\mathbb{C}((z)) / \mathbb{C}}^{1}(\mathbb{C}((z)), \mathbb{C}((z)))$ with $\left[\tilde{L}_{i}, \tilde{L}_{j}\right]=(i-j) \tilde{L}_{i+j}$, find $U \subset \mathbb{C}((z))$ such that the following three conditions are satisfied:

(i') $U \in \operatorname{Gr}(\mathbb{C}((z)))($ KP hierarchy $)$, 
(ii') $z^{-2} U \subset U$,

(iii') $\tilde{L}_{k} U \subseteq U$ for $k \geq-1$,

which are the translations of items (i)-(iii) above. Once this problem has been successfully solved, we associate with the subalgebra the $\tau$-function of the subspace $U, \tau_{U}(t)$, which, by construction, will solve KdV and Virasoro simultaneously.

Let us sketch the main steps of this construction. Our first task is concerned with the study of Witt algebras. More explicitly, let $\mathcal{W}$ be the Witt algebra; that is, the $\mathbb{C}$-vector space with basis $\left\{L_{k}\right\}_{k \in \mathbb{Z}}$ endowed with the Lie bracket $\left[L_{i}, L_{j}\right]=(i-j) L_{i+j}$, and let $\mathcal{W}^{+}$be the subalgebra generated by $\left\{L_{k}\right\}_{k \geq-1}$. Then, Section 2 is fully devoted to the study of the maps of Lie algebras

$$
\rho: \mathcal{W}^{+} \longrightarrow \mathcal{D}^{1}
$$

where $\left.\mathcal{D}^{1}:=\mathcal{D}_{\mathbb{C}((z)) / \mathbb{C}}^{1}(\mathbb{C}((z)), \mathbb{C}((z)))\right)$ denotes the algebra of first-order differential operators of $\mathbb{C}((z))$. Then, the Virasoro constraints will be written down in terms of the operators $\tilde{L}_{k}:=\rho\left(L_{k}\right)$. Some results of this section deserve special attention. For instance, Theorem 2.1 deals with explicit expressions for $\rho$; it claims that there is a 1-1-correspondence:

$$
\left\{\begin{array}{c}
\rho \in \operatorname{Hom}_{\text {Lie-alg }}\left(\mathcal{W}^{+}, \mathcal{D}^{1}\right) \\
\text { s.t. } \rho \neq 0
\end{array}\right\} \stackrel{1-1}{\longleftrightarrow}\left\{\begin{array}{c}
(h(z), c, b(z)) \text { s.t. } h^{\prime}(z) \in \mathbb{C}((z))^{*} \\
c \in \mathbb{C}, b(z) \in \mathbb{C}((z))
\end{array}\right\}
$$

which is explicitly given by

$$
\rho\left(L_{i}\right)=\frac{-h(z)^{i+1}}{h^{\prime}(z)} \partial_{z}-(i+1) c \cdot h(z)^{i}+\frac{h(z)^{i+1}}{h^{\prime}(z)} b(z) .
$$

Other results are concerned with the classification of $\operatorname{Im} \rho$ as a subalgebra of $\mathcal{D}^{1}$ (Theorem 2.7), with its conjugacy class (Theorem 2.9) as well as with the isomorphism classes of its central extensions (Theorem 2.14).

The second main problem to be tackled is the explicit description of the vector space $U$, which is the main topic in Section 3. To begin with, we examine what the stabilizer of a subspace stable under $\rho$ looks like, and, since it turns out to be $\mathbb{C}[h(z)]$ (Theorem 3.1), we conclude that, for the goals of this paper, we may restrict our study to the case of those maps $\rho$ with $h(z)=z^{-2}$ under the correspondence (1.2). Now, let us comment on a technical issue which is crucial in our approach. Observe that for any 1-dimensional $\mathbb{C}((z))$-vector space $V$, it holds that the bosonization isomorphism establishes an identification $H^{0}(\operatorname{Gr}(V)$, Det* $)=\Lambda^{\frac{\infty}{2}} V \simeq$ $\mathbb{C}\left[\left[t_{1}, t_{2}, \ldots\right]\right]$ that is compatible with the actions of the Virasoro algebra on $\Lambda^{\frac{\infty}{2}} V$ and that of the Witt algebra on $\operatorname{Gr}(V)$. Thus, instead of $\mathbb{C}((z))$ we may use any 1-dimensional $\mathbb{C}((z))$-vector space $V$. Note that replacing one vector space by another amounts to conjugating the operators. Regarding $\tau$-functions, which are our object of interest, observe that two subpaces $U_{1} \in \operatorname{Gr}\left(V_{1}\right)$ and $U_{2} \in \operatorname{Gr}\left(V_{2}\right)$ will share the same $\tau$-function as long as there is an isomorphism $V_{1} \stackrel{\sim}{\rightarrow} V_{2}$ mapping $U_{1}$ to $U_{2}$. An explanation for this lies in the property that the $\tau$-function of a point $U$ depends only on the coordinates of vectors of $U$ w.r.t. a basis of $V$ and not on $V$ or $U$ themselves. Thus, $(\mathbb{C}((z)), \rho)$ can be replaced by another 1-dimensional $\mathbb{C}((z))$-vector space endowed with the action induced by $\rho$ by conjugation.

Then, it will be proved that, for each solution $w(z)$ of the Airy equation, there is a pair $\left(V^{w v}, \rho^{w v}\right)$ as above, where $v$ depends only on $\rho$, and a subspace $\mathcal{U}(w)$ of $V^{w v}$ satisfying $\mathcal{U}(w) \in$ $\operatorname{Gr}\left(V^{w v}\right), z^{-2} \mathcal{U}(w) \subset \mathcal{U}(w)$ and $\rho^{w v}\left(L_{k}\right) \mathcal{U}(w) \subseteq \mathcal{U}(w)$. It follows that $\tau_{\mathcal{U}(w)}(t) \in \mathbb{C}\left[\left[t_{1}, t_{3}, \ldots\right]\right]$ is the desired function that solves $\mathrm{KdV}$ and Virasoro simultaneously. This is the main result of the paper (Theorem 3.12). 
Once the function has been found, we may wonder about the dependence on the choice of $w$ and whether there is more than one map producing the same function. The answer is that $\partial_{t_{2 i+1}} \partial_{t_{2 j+1}} \log \tau_{\mathcal{U}(w)}(t)$, for $i, j \geq 0$, does not depend on the $w$ chosen (Theorem 3.16). Further, the following result on uniqueness holds: let $\rho_{1}$ and $\rho_{2}$ be given and let $\tau_{1}(t)$ and $\tau_{2}(t)$ be two solutions of the KdV hierarchy and of the Virasoro constraints attached to $\rho_{1}$ and to $\rho_{2}$; then, the second derivatives of their logarithms coincide if and only if the Virasoro constraints for $\rho_{1}$ and $\rho_{2}$ coincide (Theorem 3.17). In a simpler way, the theory (i.e., KdV plus Virasoro) determines uniquely the $\tau$-function and conversely.

In the last section (see Section 4) some applications are offered. First, our results are tested against the well known case of $2 \mathrm{D}$ gravity. Using [8, 9, 17, 21, 23, 25] as the guidelines of the theory, we shall check that our formalism fits perfectly within the algebraic manipulation of the Virasoro algebra appearing in the $2 \mathrm{D}$ gravity. In particular, the fact that the partition function satisfies KdV and Virasoro follows from the very construction and does not need further proof.

A salient by-product emerge from a closer analysis of the subspace $U$ and whose study deserves future research. First in Section 4.2, Krichever theory allows us to give an alternative description of $U$ in terms of algebraic curves. Namely, under the hypotheses of Theorem 3.12, it holds that $U$ is defined by a 2:1-cover $X \rightarrow \mathbb{P}_{1}$ together with a rank 1 torsion free sheaf on $X$. Moreover, certain variations of the curve would be governed by the Painlevé equations and correspond to isomonodromic deformations. Second, one can easily check that the pair $(\mathbb{C}((z)), \rho)$ defines a $\operatorname{Gl}(n)$-oper on the punctured disk, $\operatorname{Spec} \mathbb{C}\left(\left(h(z)^{-1}\right)\right)$. We hope that our construction might be better understood within the framework of Frenkel's approach to the geometric Langlands program [14].

On the other hand, the pair $(\mathbb{C}((z)), \rho)$ defines a $\operatorname{Gl}(n)$-oper on the punctured disk, Spec $\mathbb{C}\left(\left(h(z)^{-1}\right)\right)$. Section 4.3 is concerned with this construction which is the $D$ arrow of the above diagram for $h(z)=z^{-2}$. It would be natural to expect a generalization of the relation between actions of a formal loop group (see Corollary 2.2) and opers on the punctured disk and, therefore, we believe that our construction might be better understood within the framework of Frenkel's approach to the geometric Langlands program [14].

Finally, we show that the families of Virasoro algebras used in [23, 27, 29, 30, 32, 33] for the study of the topological recursion also fit into our framework and have a natural geometrical interpretation (see Section 4.4). In particular, it's shown that a family of $\tau$-functions satisfying $\mathrm{KdV}$ and Virasoro is equivalent to a family of actions of the Witt algebra. It is worth noticing that there are instances of such 1-parameter families (e.g., the sine curve in [32]) which are indeed the spectral curve in Eynard-Orantin theory.

Before finishing this introduction, let us mention some problems that, in our opinion, can be studied with our techniques. For instance, regarding Theorem 3.17, we point out that these functions arise in the study of monodromy-preserving deformations as well as in the theory of Frobenius manifolds (e.g., [10] and [17, Section 8]). Second, the families of actions given in Section 4.4 suggests the possibility of connecting our approach with the computation of the $\tau$-function as a matrix integral (e.g., [1]). Finally, we shall extend our formalism to include special second-order differential operators in order to study loop equations and cut-and-join operators and to consider the case of the $n$-KdV hierarchy.

\section{Actions of Witt algebras}

Let $\mathcal{W}$ be the Witt algebra; that is, the Lie algebra that is freely generated by $\left\{L_{k} \mid k \in \mathbb{Z}\right\}$ as a $\mathbb{C}$-vector space and endowed with the following Lie bracket $\left[L_{i}, L_{j}\right]=(i-j) L_{i+j}$. Let us denote by $\mathcal{W}^{+} \subset \mathcal{W}$ the Lie subalgebra generated by $\left\{L_{k} \mid k \geq-1\right\}$. 
Let $V$ denote a 1-dimensional $\mathbb{C}((z))$-vector space and let $\mathcal{D}_{\mathbb{C}((z)) / \mathbb{C}}^{1}(V, V)$ be the Lie algebra generated by first-order differential operators. The symbol map is

$$
\sigma: \mathcal{D}_{\mathbb{C}((z)) / \mathbb{C}}^{1}(V, V) \longrightarrow \operatorname{Der}_{\mathbb{C}}(\mathbb{C}((z)))=\mathbb{C}((z)) \partial_{z} \stackrel{\sim}{\rightarrow} \mathbb{C}((z)),
$$

where the last map sends $f(z) \partial_{z}$ to $f(z)$.

\subsection{General form}

We are interested in pairs $(V, \rho)$ consisting of a 1-dimensional $\mathbb{C}((z))$-vector space, $V$, and a Lie algebra homomorphism:

$$
\mathcal{W}^{+} \stackrel{\rho}{\longrightarrow} \mathcal{D}_{\mathbb{C}((z)) / \mathbb{C}}^{1}(V, V) .
$$

For the sake of brevity and when no confusion arises, the pair $(V, \rho)$ will be called an action of $\mathcal{W}^{+}$.

In this subsection we shall focus on the case of $V=\mathbb{C}((z))$. Nevertheless, similar results can be proved for $V$ arbitrary by fixing an isomorphism $V \simeq \mathbb{C}((z))$ (see Section 2.2 for the dependence on the choice of the isomorphism).

Although a lot of properties on $\mathcal{W}$-modules are known, the following explicit description of its actions on $\mathcal{D}_{\mathbb{C}((z)) / \mathbb{C}}^{1}(\mathbb{C}((z)), \mathbb{C}((z)))$ seems to be brand new (see also Remark 2.15) except for the result of Kirillov when studying discrete series of unirreps for Virasoro in terms of univalent functions [24].

Theorem 2.1. Let $V$ be $\mathbb{C}((z))$ and $\rho: \mathcal{W}^{+} \rightarrow \mathcal{D}_{\mathbb{C}((z)) / \mathbb{C}}^{1}(V, V)$ be a $\mathbb{C}$-linear map such that $\rho \neq 0$. Then, the map $\rho$ is a Lie algebra homomorphism if and only if there exist functions $h(z), b(z) \in \mathbb{C}((z))$ and a constant $c \in \mathbb{C}$ such that $h^{\prime}(z) \neq 0$ and

$$
\rho\left(L_{i}\right)=\frac{-h(z)^{i+1}}{h^{\prime}(z)} \partial_{z}-(i+1) \operatorname{ch}(z)^{i}+\frac{h(z)^{i+1}}{h^{\prime}(z)} b(z) .
$$

Proof. The converse is a straightforward computation. Let us prove the direct one.

Let us write $\rho\left(L_{k}\right)=a_{k}(z) \partial_{z}+b_{k}(z)$. Since $\rho$ is a map of Lie algebras, the expression of the bracket $\left[L_{i}, L_{j}\right]=(i-j) L_{i+j}$ implies the following set of equations

$$
\begin{aligned}
& a_{i}(z) a_{j}^{\prime}(z)-a_{j}(z) a_{i}^{\prime}(z)=(i-j) a_{i+j}(z), \\
& a_{i}(z) b_{j}^{\prime}(z)-a_{j}(z) b_{i}^{\prime}(z)=(i-j) b_{i+j}(z) .
\end{aligned}
$$

Observe that if $a_{-1}(z)=\sigma\left(\rho\left(L_{-1}\right)\right)=0$, then we let $i$ be equal to -1 in equation (2.2) and have that $a_{j}(z)=0$ for all $j \geq-1$. Substituting in equation (2.3), it follows that $\rho \equiv 0$.

Hence, we now assume that $a_{-1}(z)=\sigma\left(\rho\left(L_{-1}\right)\right) \neq 0$. Let us fix $L_{-1}$ and solve this system in terms of its coefficients.

Letting $i=-1$ in equation (2.2), dividing by $a_{-1}(z)^{2}$ and integrating, it follows that $a_{j}(z)=$ $-(1+j) a_{-1}(z) \int^{z} a_{j-1}(t) a_{-1}(t)^{-2} \mathrm{~d} t$. Hence, $a_{j}(z)$ can be determined recursively from $a_{-1}(z)$. Indeed, the case $j=0$ yields $a_{0}(z)=a_{-1}(z)\left(\alpha-\int^{z} \frac{\mathrm{d} t}{a_{-1}(t)}\right)$ for $\alpha \in \mathbb{C}$. Since $a_{-1}(z), a_{0}(z) \in$ $\mathbb{C}((z))$, it follows that $\left(\alpha-\int^{z} \frac{\mathrm{d} t}{a_{-1}(t)}\right)$ must lie in $\mathbb{C}((z))$; i.e., there exists $h(z) \in \mathbb{C}((z))$ such that

$$
a_{-1}(z)=\frac{-1}{h^{\prime}(z)}
$$

Thus, setting the free term of $h(z)$ to be equal to that constant, it follows that

$$
a_{0}(z)=\frac{-h(z)}{h^{\prime}(z)} .
$$


Now, induction procedure proves straightforwardly that

$$
a_{i}(z)=\frac{-h(z)^{i+1}}{h^{\prime}(z)} .
$$

Let us now focus on the $b_{i}$ variables. Firstly, let us deal with the case $h(z)=z^{n}$; hence, $a_{i}(z)=-\frac{1}{n} z^{n i+1}$ and equation (2.3) has the following shape

$$
-\frac{1}{n} z^{n i+1} b_{j}^{\prime}(z)+\frac{1}{n} z^{n j+1} b_{i}^{\prime}(z)=(i-j) b_{i+j}(z) .
$$

Let us write $b_{j}(z)$ as $\sum_{k} b_{j, k} z^{k}$, where $b_{j, k}=0$ for $k \ll 0$. Computing the coefficients of $z^{k}$, in equation (2.3) one has the relation

$$
\left(-\frac{1}{n}(k-n i) b_{j, k-n i}+\frac{1}{n}(k-n j) b_{i, k-n j}\right)=(i-j) b_{i+j, k} .
$$

The case $j=0$ implies that $(k-n i) b_{i, k}=(k-n i) b_{0, k-n i}$ and, therefore $b_{i, k}=b_{0, k-n i}$ for $k \neq n i$; that is, the difference between $z^{-n i} b_{i}(z)$ and $b_{0}(z)$ is a constant. Expressing this condition in terms of $b_{-1}(z)$, the following formula for $b_{i}(z)$ holds

$$
b_{i}(z)=\left(c_{i}+b_{-1}(z) z^{n}\right) z^{n i}
$$

for some $c_{i} \in \mathbb{C}$ and $c_{-1}=0$. Plugging this into equation (2.3) and setting $i$ equal to -1 , we find a constraint for the $c_{i}$

$$
j c_{j}+c_{-1}-(j+1) c_{j-1}=0, \quad c_{-1}=0,
$$

whose general solution is

$$
c_{j}=-c \cdot(j+1)
$$

for a complex number $c=-c_{0} \in \mathbb{C}$. Bearing in mind that $h^{\prime}(z)$ is invertible, there is no harm in assuming that $b_{-1}(z)$ is of the form $\frac{1}{h^{\prime}(z)} b(z)$. Thus, from equations $(2.5)$ and (2.6), the general solution for the case $h(z)=z^{n}$ is

$$
b_{i}(z)=\left(-(i+1) c+z^{n} \frac{b(z)}{n z^{n-1}}\right) z^{n i} .
$$

The general case, i.e., for $h(z)$ arbitrary, follows from the fact that there is a $\mathbb{C}$-algebra automorphism of $\mathbb{C}[[z]], \phi$, such that $\phi(h(z))=z^{n}$ where $h(z)=a_{n} z^{n}+a_{n+1} z^{n+1}+\cdots$ and $a_{n} \neq 0$. That is, in order to solve equation (2.3), we consider $\phi$, such that $\phi(h(z))=z^{n}$. We transform equation (2.3) by $\phi$, which is equation (2.4), and consider its solutions (2.7). Thus, transforming the solutions by the inverse automorphism, $\phi^{-1}$, we have that the general solution for equation (2.3) is as follows

$$
b_{i}(z)=\left(-(i+1) c+h(z) \frac{b(z)}{h^{\prime}(z)}\right) h(z)^{i} .
$$

Observing that in the previous proof only the operators $\rho\left(L_{i}\right)$ for $i=-1,0$ were necessary, we have the following:

Corollary 2.2. The restriction maps

$$
\mathfrak{s l}_{2}(\mathbb{C}) \simeq\left\langle L_{-1}, L_{0}, L_{1}\right\rangle \subset \mathcal{W}^{+} \subset \mathcal{W}
$$

induce isomorphisms

$$
\operatorname{Hom}_{\text {Lie-alg }}\left(\mathcal{W}, \mathcal{D}^{1}\right) \stackrel{\sim}{\rightarrow} \operatorname{Hom}_{\text {Lie-alg }}\left(\mathcal{W}^{+}, \mathcal{D}^{1}\right) \stackrel{\sim}{\rightarrow} \operatorname{Hom}_{\text {Lie-alg }}\left(\mathfrak{s l}_{2}(\mathbb{C}), \mathcal{D}^{1}\right),
$$

where $\mathcal{D}^{1}$ denotes $\mathcal{D}_{\mathbb{C}((z)) / \mathbb{C}}^{1}(\mathbb{C}((z)), \mathbb{C}((z)))$. Under these isomorphisms, the $\mathfrak{s l}_{2}(\mathbb{C})$ representation associated to $\rho$ (attached to $(h(z), c, b(z)))$ has Casimir operator $1+4 c^{2}$. 
Remark 2.3. The combination of Corollary 2.2 and formula $(2.1)$ with $h(z)=z^{-1}+d$ for $d \in \mathbb{C}$ agrees with Mulase's result on injective Lie algebra morphisms from $\mathfrak{s l}_{2}(\mathbb{C})$ to $\mathcal{D}^{1}[31$, Proposition 8.6].

Remark 2.4. The reason for restricting ourselves to the case of first-order differential operators of $V$ instead of more general operators relies on the fact that this is the case in most situations, e.g., 2D gravity. From a more theoretical point of view, the equivalence of categories between Atiyah algebras and differential operator algebras [5], means that $\mathcal{D}_{\mathbb{C}((z)) / \mathbb{C}}^{1}(V, V)$ is the natural candidate to begin with. Consequently, one expect a link with he moduli space of pairs consisting of a curve and a line bundle on it, since the Lie group associated with $\mathcal{D}_{\mathbb{C}((z)) / \mathbb{C}}^{1}(V, V)$ uniformizes this moduli space [18]. By studying higher order differential operators, one may relate this with the study $W$-algebras (and their representation theory, etc. [16], [1, Section 1.2]).

Remark 2.5. It holds that

$$
\left[\rho\left(L_{k}\right), h(z)^{j}\right]=-j \cdot h(z)^{k+j}
$$

as $\mathbb{C}$-linear operators on $V$. For $g(z) \in \mathbb{C}((z))$ arbitrary, it holds that $\left[\rho\left(L_{k}\right), g\right]=-\frac{h(z)^{k+1}}{h^{\prime}(z)} g^{\prime}(z)$.

Corollary 2.6. If $\sigma\left(\rho\left(L_{-1}\right)\right) \neq 0$, then $\rho$ and $\sigma \circ \rho$ are injective and $\operatorname{Im}(\sigma \circ \rho)=\frac{1}{h^{\prime}(z)} \mathbb{C}[h(z)]$.

Proof. Let us prove that $\sigma \circ \rho$ is injective. Let us assume that a linear combination $\sum_{k} \lambda_{k} L_{k}$ lies on the kernel of $\sigma \circ \rho$; that is, $\sigma\left(\rho\left(\sum_{k} \lambda_{k} L_{k}\right)\right)=0$. The previous Theorem yields the identity $\sigma\left(\rho\left(L_{k}\right)\right)=-\frac{h(z)^{k+1}}{h^{\prime}(z)}$, and therefore we have that

$$
0=\sigma\left(\rho\left(\sum_{k} \lambda_{k} L_{k}\right)\right)=\left(\sum_{k} \lambda_{k} h(z)^{k+1}\right) \sigma\left(\rho\left(L_{-1}\right)\right) .
$$

Since $\sigma\left(\rho\left(L_{-1}\right)\right)=-\frac{1}{h^{\prime}(z)} \neq 0$, it follows that $\lambda_{k}=0$ for all $k$, and the claim is proved.

Second, the injectivity of $\sigma \circ \rho$ implies the injectivity of $\rho$. Finally, $\operatorname{Im}(\sigma \circ \rho)=\frac{1}{h^{\prime}(z)} \mathbb{C}[h(z)]$ follows easily from equation (2.1).

Let $\mathfrak{v}: \mathbb{C}((z)) \rightarrow \mathbb{Z} \cup\{\infty\}$ be the valuation associated with $z$; that is, $\mathfrak{v}(0)=\infty$ and, for $h(z) \neq 0, \mathfrak{v}(h(z))=a$ iff $a$ is the largest integer number such that $h(z) \in z^{a} \mathbb{C}[[z]]$ and, in this situation, $a$ will be called the order of $h$.

Theorem 2.7. For $i=1,2$, let $h_{i}(z), b_{i}(z) \in \mathbb{C}((z))$ and $c_{i} \in \mathbb{C}$ with $h_{i}^{\prime}(z) \neq 0$. Let $(V=$ $\left.\mathbb{C}((z)), \rho_{i}\right)$ be the action of $\mathcal{W}^{+}$associated with elements $h_{i}(z), b_{i}(z), c_{i}$, as in Theorem 2.1 .

If $\operatorname{Im} \rho_{1}=\operatorname{Im} \rho_{2}$ and the signs of $\mathfrak{v}\left(h_{1}(z)\right)$ and $\mathfrak{v}\left(h_{2}(z)\right)$ are equal and negative, then $b_{1}(z)=$ $b_{2}(z), c_{1}=c_{2}$ and $h_{1}(z)=\alpha h_{2}(z)+\beta$ for some $\alpha \in \mathbb{C}^{*}, \beta \in \mathbb{C}$.

Conversely, if $b_{1}(z)=b_{2}(z), c_{1}=c_{2}$ and $h_{1}(z)=\alpha h_{2}(z)+\beta$ for some $\alpha \in \mathbb{C}^{*}, \beta \in \mathbb{C}$, then $\operatorname{Im} \rho_{1}=\operatorname{Im} \rho_{2}$. Moreover, there exists a Lie algebra automorphism $\phi$ of $\mathcal{W}^{+}$such that $\rho_{2}=\rho_{1} \circ \phi$.

Proof. From the hypothesis $\operatorname{Im} \rho_{1}=\operatorname{Im} \rho_{2}$, it holds that there exist $\left\{\lambda_{k l} \mid k, l \geq-1\right\}$ such that

$$
\rho_{1}\left(L_{k}\right)=\sum_{l \geq-1} \lambda_{k l} \rho_{2}\left(L_{l}\right)
$$

By the explicit expression obtained in Theorem 2.1, this identity is equivalent to the equations

$$
\frac{h_{1}(z)^{k+1}}{h_{1}^{\prime}(z)}=\sum_{l \geq-1} \lambda_{k l} \frac{h_{2}(z)^{l+1}}{h_{2}^{\prime}(z)}
$$


and

$$
\frac{h_{1}(z)^{k+1}}{h_{1}^{\prime}(z)} b_{1}(z)-(k+1) c_{1} h_{1}(z)^{k}=\sum_{l \geq-1} \lambda_{k l}\left(\frac{h_{2}(z)^{l+1}}{h_{2}^{\prime}(z)} b_{2}(z)-(l+1) c_{2} h_{2}(z)^{l}\right) .
$$

Observe that the derivative of equation (2.9) w.r.t. $z$ yields

$$
\begin{aligned}
(k+1) h_{1}(z)^{k}-\frac{h_{1}(z)^{k+1} h_{1}^{\prime \prime}(z)}{h_{1}^{\prime}(z)^{2}} & =\sum_{l \geq-1} \lambda_{k l}\left((l+1) h_{2}(z)^{l}-\frac{h_{2}(z)^{l+1} h_{2}^{\prime \prime}(z)}{h_{2}^{\prime}(z)^{2}}\right) \\
& =\sum_{l \geq-1} \lambda_{k l}(l+1) h_{2}(z)^{l}-\frac{h_{1}(z)^{k+1}}{h_{1}^{\prime}(z)} \cdot \frac{h_{2}^{\prime \prime}(z)}{h_{2}^{\prime}(z)}
\end{aligned}
$$

One computes equation (2.10) plus equation (2.9) times $\left(-b_{2}(z)\right)$ plus equation $(2.11)$ multiplied by $c_{2}$, and one obtains

$$
\frac{h_{1}(z)^{k+1}}{h_{1}^{\prime}(z)}\left(\left(b_{1}(z)-b_{2}(z)\right)-(k+1)\left(c_{1}-c_{2}\right) \frac{h_{1}^{\prime}(z)}{h_{1}(z)}-\left(\frac{h_{1}^{\prime \prime}(z)}{h_{1}^{\prime}(z)}-\frac{h_{2}^{\prime \prime}(z)}{h_{2}^{\prime}(z)}\right) c_{2}\right)=0 .
$$

Since this holds for all $k \geq-1$, it follows that

$$
c_{1}-c_{2}=0, \quad\left(b_{1}(z)-b_{2}(z)\right)-\left(\frac{h_{1}^{\prime \prime}(z)}{h_{1}^{\prime}(z)}-\frac{h_{2}^{\prime \prime}(z)}{h_{2}^{\prime}(z)}\right) c_{2}=0 .
$$

Hence $c_{1}=c_{2}$.

Further, Corollary 2.6 shows that $\frac{1}{h_{1}^{\prime}(z)} \mathbb{C}\left[h_{1}(z)\right]=\frac{1}{h_{2}^{\prime}(z)} \mathbb{C}\left[h_{2}(z)\right]$. Hence, there are polynomials $p_{i}$ such that $\frac{1}{h_{1}^{\prime}(z)}=\frac{p_{2}\left(h_{2}(z)\right)}{h_{2}^{\prime}(z)}$ and $\frac{1}{h_{2}^{\prime}(z)}=\frac{p_{1}\left(h_{1}(z)\right)}{h_{1}^{\prime}(z)}$. These identities imply that

$$
p_{1}\left(h_{1}(z)\right) p_{2}\left(h_{2}(z)\right)=1
$$

and, thus

$$
\operatorname{deg}\left(p_{1}\right) \mathfrak{v}\left(h_{1}(z)\right)+\operatorname{deg}\left(p_{2}\right) \mathfrak{v}\left(h_{2}(z)\right)=0 .
$$

The assumption about the signs of $\mathfrak{v}\left(h_{i}(z)\right)$ implies that $p_{i}$ is constant for $i=1,2$, say $p_{1}(x)=$ $\alpha \in \mathbb{C}^{*}$. And, therefore, $h_{1}^{\prime}(z)=\alpha h_{2}^{\prime}(z)$, so that there exists $\beta \in \mathbb{C}$ with $h_{1}(z)=\alpha h_{2}(z)+\beta$.

Finally, substituting in equation (2.12), one has that $b_{1}(z)=b_{2}(z)$.

Let us now prove the converse. Using the formula (2.1), it is straightforwardly checked that

$$
\begin{aligned}
& \rho_{2}\left(L_{-1}\right)=\frac{1}{\alpha} \rho_{1}\left(L_{-1}\right), \\
& \rho_{2}\left(L_{i}\right)=h_{2}(z)^{i}\left(\frac{h_{2}(z)}{\alpha} \rho_{1}\left(L_{-1}\right)-(i+1) c\right) \quad \forall i \geq-1 .
\end{aligned}
$$

Our first task is to prove that $\rho_{2}\left(L_{i}\right)$ is a linear combination of $\left\{\rho_{1}\left(L_{k}\right)\right\}$. Let us compute $\rho_{2}\left(L_{i}\right)$

$$
\begin{aligned}
\rho_{2}\left(L_{i}\right) & =\left(\alpha h_{1}(z)+\beta\right)^{i}\left(\frac{\alpha h_{1}(z)+\beta}{\alpha} \rho_{1}\left(L_{-1}\right)-(i+1) c\right) \\
& =\alpha^{i}\left(h_{1}(z)+\frac{\beta}{\alpha}\right)^{i}\left(h_{1}(z) \rho_{1}\left(L_{-1}\right)+\frac{\beta}{\alpha} \rho_{1}\left(L_{-1}\right)-(i+1) c\right) \\
& =\alpha^{i}\left(\sum_{j=0}^{i}\left(\begin{array}{l}
i \\
j
\end{array}\right) h_{1}(z)^{j}\left(\frac{\beta}{\alpha}\right)^{i-j}\right)\left(h_{1}(z) \rho_{1}\left(L_{-1}\right)+\frac{\beta}{\alpha} \rho_{1}\left(L_{-1}\right)-(j+1) c+(j-i) c\right)
\end{aligned}
$$




$$
\begin{aligned}
& =\alpha^{i} \sum_{j=0}^{i}\left(\begin{array}{l}
i \\
j
\end{array}\right)\left(\frac{\beta}{\alpha}\right)^{i-j}\left(\rho_{1}\left(L_{j}\right)+h_{1}(z)^{j}\left(\frac{\beta}{\alpha} \rho_{1}\left(L_{-1}\right)(j-i) c\right)\right) \\
& =\alpha^{i} \sum_{j=0}^{i}\left(\begin{array}{l}
i \\
j
\end{array}\right)\left(\frac{\beta}{\alpha}\right)^{i-j}\left(\rho_{1}\left(L_{j}\right)+\frac{\beta}{\alpha}\left(\rho_{1}\left(L_{j-1}\right)+j c h_{1}(z)^{j-1}\right)+(j-i) \operatorname{ch}_{1}(z)^{j}\right) .
\end{aligned}
$$

Bearing in mind that the term

$$
\sum_{j=0}^{i}\left(\begin{array}{l}
i \\
j
\end{array}\right)\left(\frac{\beta}{\alpha}\right)^{i-j}\left(\frac{\beta}{\alpha}\left(j c h_{1}(z)^{j-1}\right)+(j-i) \operatorname{ch}_{1}(z)^{j}\right)
$$

vanishes identically, the above expression yields

$$
\begin{aligned}
\rho_{2}\left(L_{i}\right) & =\alpha^{i} \sum_{j=0}^{i}\left(\begin{array}{l}
i \\
j
\end{array}\right)\left(\frac{\beta}{\alpha}\right)^{i-j}\left(\rho_{1}\left(L_{j}\right)+\frac{\beta}{\alpha} \rho_{1}\left(L_{j-1}\right)\right) \\
& =\left(\frac{\beta}{\alpha}\right)^{i+1} \rho_{1}\left(L_{-1}\right)+\sum_{j=0}^{i-1}\left(\left(\begin{array}{l}
i \\
j
\end{array}\right)+\left(\begin{array}{c}
i \\
j+1
\end{array}\right)\right)\left(\frac{\beta}{\alpha}\right)^{i-j} \rho_{1}\left(L_{j}\right)+\rho_{1}\left(L_{i}\right) .
\end{aligned}
$$

This explicit expression shows at once that $\operatorname{Im} \rho_{2}=\operatorname{Im} \rho_{1}$.

Finally, consider

$$
\phi\left(L_{i}\right):=\left(\frac{\beta}{\alpha}\right)^{i+1} L_{-1}+\sum_{j=0}^{i-1}\left(\begin{array}{c}
i+1 \\
j+1
\end{array}\right)\left(\frac{\beta}{\alpha}\right)^{i-j} L_{j}+L_{i} .
$$

The fact that $\phi=\left(\left.\rho_{1}\right|_{\operatorname{Im} \rho_{1}}\right)^{-1} \circ \rho_{2}$ implies that $\phi$ is a Lie algebra automorphism of $\mathcal{W}^{+}$and that $\rho_{2}=\rho_{1} \circ \phi$.

Example 2.8 ( $m$-reduced KP hierarchy). In particular, it is easy to check that given an action $(V, \rho)$ and a non-zero integer number $m$, the map $\rho_{m}\left(L_{k}\right):=\frac{1}{m} \rho\left(L_{m k}\right)$ gives rise to another action of $\mathcal{W}^{+}$on $V$

$$
\begin{aligned}
{\left[\rho_{m}\left(L_{i}\right), \rho_{m}\left(L_{j}\right)\right] } & =\frac{1}{m^{2}}\left[\rho\left(L_{m i}\right), \rho\left(L_{m j}\right)\right]=\frac{1}{m^{2}}(m i-m j) \rho\left(L_{m(i+j)}\right) \\
& =(i-j) \frac{1}{m} \rho\left(L_{m(i+j)}\right)=(i-j) \rho_{m}\left(L_{i+j}\right) .
\end{aligned}
$$

Moreover, an easy computation yields

$$
\rho_{m}\left(L_{k}\right)=\frac{1}{m} \rho\left(L_{m k}\right)=-\frac{h(z)^{m k+1}}{m h^{\prime}(z)} \partial_{z}-\frac{1}{m}(m k+1) \operatorname{ch}(z)^{m k}+\frac{h(z)^{m k+1}}{m h^{\prime}(z)} b(z) .
$$

Thus, this action corresponds to the data $\tilde{h}(z)=h(z)^{m}, \tilde{c}=c$ and $\tilde{b}(z)=(1-m) c \frac{h^{\prime}(z)}{h(z)}+b(z)$.

\subsection{Conjugation}

Let us now introduce a generalization of the notion of conjugated action that will be needed later on.

Let us begin with an example. Assume we have a differential equation $P \psi(z)=0$ and we want to solve it by virtue of a replacement $\psi(z)=v(z) \phi(z)$, for a given function $v(z)$; that is, we shall solve $P(v(z) \phi(z))=0$ for an unknown function $\phi(z)$. This is equivalent to solving $\left(v(z)^{-1} \circ P \circ v(z)\right) \phi(z)=0$, where $v(z)$ is regarded as an operator; namely, the homothety of 
ratio $v(z)$. For instance, if $P$ is a first-order differential operator with symbol $\sigma(P)$, it holds that

$$
v(z)^{-1}(P \psi(z))=\left(v(z)^{-1} \circ P \circ v(z)\right) \phi(z)=\left(P+\sigma(P) \frac{v^{\prime}(z)}{v(z)}\right) \phi(z) .
$$

Therefore, solving the differential equation $P \psi(z)=0$ is equivalent to solving

$$
\left(P+\sigma(P) \frac{v^{\prime}(z)}{v(z)}\right) \phi(z)=0 .
$$

Observe that, from the point of view of analysis, the space of functions in which $\psi$ and $\phi$ lie might be different (e.g., different convergence domains, etc.). In particular, conjugation is an instance of gauge transformation.

Let us recall from [18] the definition of the group of semilinear transformations and some of its properties. The group of semilinear transformations of a finite-dimensional $\mathbb{C}((z))$-vector space $V$, denoted by $\operatorname{SGl}_{\mathbb{C}((z))}(V)$, consists of $\mathbb{C}$-linear automorphisms $\gamma: V \rightarrow V$ such that there exists a $\mathbb{C}$-algebra automorphism of $\mathbb{C}((z)), g$, satisfying

$$
\gamma(f(z) \cdot v)=g(f(z)) \cdot \gamma(v) \quad \forall f(z) \in \mathbb{C}((z)), \quad v \in V,
$$

and, therefore, $\operatorname{SGl}(\mathbb{C}((z)))=\operatorname{Aut}_{\mathbb{C} \text {-alg }} \mathbb{C}((z)) \ltimes \mathbb{C}((z))^{*}$.

The Lie algebra of $\operatorname{SGl}_{\mathbb{C}((z))}(V)$ consists of first-order differential operators on $V$ with scalar symbol, $\mathcal{D}_{\mathbb{C}((z)) / \mathbb{C}}^{1}(V, V)$, and the symbol coincides with the map induced by the group homomorphism that sends $\gamma$ to $g$ (related by equation (2.13)) between their Lie algebras.

Theorem 2.9. The space Hom $\mathrm{Hie}_{\text {alg }}\left(\mathcal{W}^{+}, \mathcal{D}^{1}\right) \backslash\{0\}$ carries an action of the group $\mathrm{SGl}(\mathbb{C}((z)))$ by conjugation and the quotient space is $\mathbb{Z} \times \mathbb{C} \times\left(\mathbb{C}((z)) / \mathbb{Z} z^{-1}+\mathbb{C}[[z]]\right)$.

Proof. Let us begin studying the action of the automorphism group $G:=\operatorname{Aut}_{\mathbb{C} \text {-alg }} \mathbb{C}((z))$ (for a study and applications of this group, see [34]). Let us denote elements of $G$ with big Greek letters $(\Phi, \Psi, \ldots)$ and, for each of them, let the corresponding small Greek letter denote the image of $z$; that is

$$
\Phi(f(z))=f(\phi(z))
$$

and observe that $\mathfrak{v}(\phi(z))=1$ in order for $\Phi$ to be an isomorphism.

We consider the action of $G$ on the space of actions by conjugation; i.e.,

$$
(\Phi, \rho) \mapsto \rho^{\Phi} \quad \text { where } \quad \rho^{\Phi}\left(L_{k}\right):=\Phi \circ \rho\left(L_{k}\right) \circ \Phi^{-1} \quad \forall k .
$$

Let us check that this definition makes sense. Let $\rho$ be given by a triple $(h(z), c, b(z))$. It is straightforward that

$$
\begin{aligned}
\rho^{\Phi}\left(L_{-1}\right) f(z) & =\left(\Phi \circ \rho\left(L_{-1}\right) \circ \Phi^{-1}\right) f(z)=\Phi\left(\left(-\frac{1}{h^{\prime}(z)} \partial_{z}+\frac{b(z)}{h^{\prime}(z)}\right) f\left(\phi^{-1}(z)\right)\right) \\
& =\left(-\frac{\left(\phi^{-1}\right)^{\prime}(\phi(z))}{h^{\prime}(\phi(z))} \partial_{z}+\frac{b(\phi(z))}{h^{\prime}(\phi(z))}\right) f(z) .
\end{aligned}
$$

Note that expanding and derivating the identity $\Phi \circ \Phi^{-1}(z)=z$, one gets that $\left(\phi^{-1}\right)^{\prime}(\phi(z))$. $\phi^{\prime}(z)=1$ and, thus

$$
\frac{\left(\phi^{-1}\right)^{\prime}(\phi(z))}{h^{\prime}(\phi(z))}=\frac{1}{\partial_{z} h(\phi(z))} .
$$


Summing up, the transformation $\Phi$ acts on triples as follows

$$
(\Phi,(h(z), c, b(z))) \mapsto(h(\phi(z)), c, b(\phi(z)))
$$

Second, we study the action of $\mathbb{C}((z))^{*}$. Bearing in mind the discussion of the beginning of this subsection, we consider the action

$$
(s(z), \rho) \mapsto\left(s(z) \circ \rho \circ s(z)^{-1}\right),
$$

so that, in terms of triples, it holds that

$$
(s(z),(h(z), c, b(z))) \mapsto\left(h(z), c, b(z)-\frac{s^{\prime}(z)}{s(z)}\right) .
$$

One checks easily that the first defined action intertwines the second one; that they yield an action of the semidirect product $G \ltimes \mathbb{C}((z))^{*}$; and that the quotient space under this action is $\mathbb{Z} \times \mathbb{C} \times\left(\mathbb{C}((z)) / \mathbb{Z} z^{-1}+\mathbb{C}[[z]]\right)$, where an action $\rho$, corresponding to a triple $(h(z), c, b(z))$ is mapped to $(\mathfrak{v}(h(z)), c, \bar{b}(z))(\bar{b}(z)$ being the equivalence class of $b(z))$.

Nevertheless, the conjugation also makes sense if $v(z)$ is replaced by any linear operator on the space of functions such that $\frac{v^{\prime}(z)}{v(z)}$ can be identified with an element in $\mathbb{C}((z))$. This is the case of the example at the beginning of this subsection, but it also holds for functions $v(z)$ admitting an asymptotic expansion at 0 . For instance, for the formal expression $v(z):=\exp \left(\int s(z) \mathrm{d} z\right)$ where $s(z) \in \mathbb{C}((z))$, the quotient $\frac{v^{\prime}(z)}{v(z)}$ will be identified with $s(z)$. Indeed, the conjugation by $\exp \left(-\frac{2}{3} z^{-3}\right)$ was used in [21] when solving a differential equation. For another example, let us consider $v(z)$ to be a solution of the second-order differential equation $v^{\prime \prime}(z)+\frac{1}{2} S(h) v(z)=0$, where $S(h)$ denotes the Schwarzian derivative of $h$, such that $\frac{v^{\prime}(z)}{v(z)} \in \mathbb{C}((z))$, which holds true in many cases (e.g., when $S(h) \in \mathbb{C}((z))$ ).

It is worth noticing that once $\frac{v^{\prime}(z)}{v(z)}$ is thought of as an element of $\mathbb{C}((z)), \frac{v^{\prime \prime}(z)}{v(z)}$ will be identified with $\left(\frac{v^{\prime}(z)}{v(z)}\right)^{2}+\left(\frac{v^{\prime}(z)}{v(z)}\right)^{\prime} \in \mathbb{C}((z))$. By abuse of notation, we define $\mathrm{d} \log v(z):=\frac{v^{\prime}(z)}{v(z)}$.

Thus, for $P \in \mathcal{D}^{1}(\mathbb{C}((z)))$ and $v(z)$ as above, we consider another first-order differential operator $P^{v} \in \mathcal{D}^{1}\left(\mathbb{C}((z)) \otimes_{\mathbb{C}} \mathbb{C} v(z)\right)$ defined by

$$
P^{v}(f(z) \otimes v(z)):=\left(\left(P+\sigma(P) \frac{v^{\prime}(z)}{v(z)}\right)(f)\right) \otimes v(z) .
$$

The induced map from $\mathcal{D}^{1}(\mathbb{C}((z)))$ to $\mathcal{D}^{1}\left(\mathbb{C}((z)) \otimes_{\mathbb{C}} \mathbb{C} v(z)\right)$ is a Lie algebra homomorphism.

Definition 2.10. The conjugated action of $(V, \rho)$ by $v(z)$ is the pair $\left(V^{v}, \rho^{v}\right)$, consisting of the 1-dimensional $\mathbb{C}((z))$-vector space $V^{v}:=V \otimes_{\mathbb{C}} \mathbb{C} v(z)$ together with the action defined by

$$
\rho^{v}\left(L_{k}\right)(f(z) \otimes v(z)):=\left(\rho\left(L_{k}\right)(f(z))+\sigma\left(\rho\left(L_{k}\right)\right) f(z) \frac{v^{\prime}(z)}{v(z)}\right) \otimes v(z) .
$$

In particular, if the data $h(z), c, b(z)$ define an action $\rho$, then $h(z), c, b(z)-\frac{v^{\prime}(z)}{v(z)}$ define $\rho^{v}$.

Remark 2.11. Our generalized notion of conjugation allows us to consider other actions of $\mathbb{C}((z))$. For instance, if we consider $s(z) \in \mathbb{C}((z))$ acting by conjugation by $\exp (s(z)$ ) (resp. $\left.\exp \left(\int s(z)\right)\right)$, then the quotient space by $G \ltimes \mathbb{C}((z))$ is $\mathbb{Z} \times \mathbb{C} \times \mathbb{C} / \mathbb{Z}$ (resp. $\mathbb{Z} \times \mathbb{C}$, which is related to Theorem 2.14). 


\subsection{Central extensions}

Recall from [3] that, given a pair $\left(V, V_{+}\right)$consisting of a $\mathbb{C}$-vector space and a subspace of it, there exists a $\mathbb{C}$-scheme whose rational points correspond to the vector subspaces $W \subseteq V$ such that

$$
W \cap V_{+} \quad \text { and } \quad V / W+V_{+}
$$

are finite-dimensional vector spaces over $\mathbb{C}$. It is called the Sato Grassmannian or infinite Grassmannian of $V$ (see [39] for an analytical approach).

Further, the group $\operatorname{SGl}(\mathbb{C}((z)))$ acts on the Sato Grassmannian of $V, \operatorname{Gr}(V)$, preserving the determinant bundle $\operatorname{Det}_{V}$. This has two significant consequences. Firstly, this group also acts on the projectivization of $H^{0}\left(\operatorname{Gr}(V)\right.$, Det $\left.V_{V}^{*}\right)$, making the Plücker embedding equivariant

$$
\operatorname{Gr}(V) \hookrightarrow \mathbb{P} H^{0}\left(\operatorname{Gr}(V), \operatorname{Det}_{V}^{*}\right)^{*} .
$$

Second, the group of automorphisms of $\operatorname{Det}_{V}$ that lift automorphisms of $\operatorname{Gr}(V)$ defined by elements of $\operatorname{SGl}_{\mathbb{C}((z))}(V)$; i.e.,

$$
\widetilde{\operatorname{SGl}}_{\mathbb{C}((z))}(V):=\left\{\begin{array}{cc}
\operatorname{Det}_{V}^{*} \stackrel{\sim}{\longrightarrow} \operatorname{Det}_{V}^{*} \\
\downarrow & \downarrow \\
\operatorname{Gr}(V) \stackrel{g}{\sim} \operatorname{Gr}(V)
\end{array} \text { where } g \in \operatorname{SGl}_{\mathbb{C}((z))}(V)\right\}
$$

defines a canonical central extension

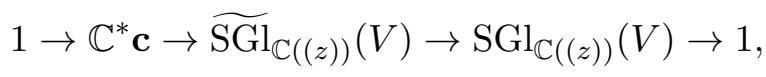

where $\mathbf{c}$ is the central element (central charge).

Now, let us assume that an action $(V, \rho)$ of $\mathcal{W}^{+}$is given. Since $\rho$ can be extended to $\mathcal{W}$, we may consider the pullback of the central extension of Lie algebras induced by (2.15) and obtain a central extension $\widetilde{\mathcal{W}}$ of $\mathcal{W}$

$$
0 \rightarrow \mathbb{C} \mathbf{c} \rightarrow \widetilde{\mathcal{W}} \rightarrow \mathcal{W} \rightarrow 0
$$

where we recall that $\widetilde{\mathcal{W}}=\mathcal{W} \oplus \mathbb{C} \mathbf{c}$, as vector spaces. Its Lie bracket will be computed below.

Remark 2.12. Recall that the multiplicative group $\mathbb{C}((z))^{*}$ acts by homotheties on $\operatorname{Gr}(V)$ and by conjugation (i.e., gauge transformation) on $\operatorname{SGl}_{\mathbb{C}((z))}(V)$. Furthermore, the action map

$$
\operatorname{SGl}_{\mathbb{C}((z))}(V) \times \operatorname{Gr}(V) \longrightarrow \operatorname{Gr}(V)
$$

is equivariant with respect to these actions. Thus, for fixed $U \in \operatorname{Gr}(V)$, the map induced by the orbit map at the level of tangent spaces

$$
T_{\mathrm{Id}} \operatorname{SGl}_{\mathbb{C}((z))}(V) \simeq \mathcal{D}_{\mathbb{C}((z)) / \mathbb{C}}^{1}(V, V) \longrightarrow T_{U} \operatorname{Gr}(V) \simeq \operatorname{Hom}(U, V / U)
$$

intertwines gauge transformations (on the l.h.s.) and KP flows (on the r.h.s.). In fact, requirements (ii') and (iii') of the introduction, $z^{-2} U \subset U$ and $\tilde{L}_{k} U \subseteq U$, should be understood at the tangent space $T_{U} \operatorname{Gr}(V)$.

In fact, once $(V, \rho)$ is given, the above discussion allows us to obtain a natural map at the level of tangent spaces

$$
\begin{aligned}
\mathcal{W}^{+} \stackrel{\rho}{\longrightarrow} \mathcal{D}_{\mathbb{C}((z)) / \mathbb{C}}^{1}(V, V) \longrightarrow & \operatorname{Hom}(U, V / U), \\
L \longmapsto & \hookrightarrow V^{\rho(L)} \stackrel{\longrightarrow}{\rightarrow} V \rightarrow V / U,
\end{aligned}
$$


where $U$ is a point in $\operatorname{Gr}(V)$ and we observe that this map can be extended to $\mathcal{W}$ and that the equivariance of the Plücker morphism yields a natural map

$$
\begin{gathered}
\widetilde{\mathcal{W}} \stackrel{\hat{\rho}}{\longrightarrow} H^{0}\left(\operatorname{Gr}(V), \operatorname{Det}_{V}^{*}\right)^{*} \stackrel{\sim}{\longrightarrow} \mathbb{C}\left[\left[t_{1}, t_{2}, \ldots\right]\right], \\
L \longmapsto \text { evaluation at } \rho(L)(U) \longmapsto \hat{\rho}(L) \tau_{U}(t)
\end{gathered}
$$

(c, being the central element, acts as a homothety).

Remark 2.13. It is worth pointing out that $H^{0}\left(\operatorname{Gr}(V)\right.$, Det $V$, also written as $\Lambda^{\frac{\infty}{2}} V$ in the literature, corresponds to the formulation in terms of fermions and that the isomorphism on the r.h.s. is the bosonization morphism (see, e.g., [20]). Observe that the rightmost term of the previous map, $\mathbb{C}\left[\left[t_{1}, t_{2}, \ldots\right]\right]$, does not depend on $V$ since the $\tau$-function of a point $U$ depends only on the coordinates of vectors of $U$ w.r.t. a basis of $V$ but not on the basis (e.g., [25, Lemma 4.2.]).

Our next task is concerned with the explicit computation of the Lie bracket of $\widetilde{\mathcal{W}}$ or, tantamount to this, with the classification of $\widehat{\mathcal{W}}$ as a central extension. Since $H^{2}(\mathcal{W}, \mathbb{C}) \simeq \mathbb{C}$ and it is generated by the cocycle of the Virasoro algebra, $\operatorname{Vir}\left(L_{r}, L_{s}\right)=\delta_{r,-s} \frac{r^{3}-r}{12}$, it follows that the 2-cocycle classifying the central extension $\widetilde{\mathcal{W}}$ has to be a multiple of Vir.

It is known that the cocycle of the extension (2.15) is the so-called Gelfand-Fuchs cocycle whose general expression reads as follows (see, for instance, [20, 19])

$$
\Psi\left(a(z) \partial_{z}^{r}, b(z) \partial_{z}^{s}\right):=\frac{r ! s !}{(r+s+1) !} \operatorname{Res}_{z=0}\left(\partial_{z}^{s+1} a(z) \partial_{z}^{r} b(z)\right) \mathrm{d} z .
$$

This means that $\widetilde{\mathcal{W}}$, generated by $\left\{L_{k}, \mathbf{c}\right\}$, carries the Lie bracket

$$
\left[L_{r}, L_{s}\right]:=(r-s) L_{r+s}+\Psi\left(L_{r}, L_{s}\right) \mathbf{c} .
$$

Theorem 2.14. Let $(V, \rho)$ be given. The isomorphism class of the 2 -cocyle of $\widetilde{\mathcal{W}}, \Psi$, is

$$
-2\left(1-6 c+6 c^{2}\right) \mathfrak{v}(h(z)) \operatorname{Vir},
$$

where $h(z)$, c are given by Theorem 2.1 .

Proof. Let us recall what a 2-coboundary looks like. A 2-cocycle

$$
\alpha: \mathcal{W} \times \mathcal{W} \rightarrow \mathbb{C}
$$

is a 2-coboundary iff there exists a linear map $f: \mathcal{W} \rightarrow \mathbb{C}$ such that

$$
\alpha\left(L, L^{\prime}\right)=f\left(\left[L, L^{\prime}\right]\right)
$$

Thus, in our case, since $\left\{L_{r} \mid r \in \mathbb{Z}\right\}$ is a basis of $\mathcal{W}$, a function $f: \mathcal{W} \rightarrow \mathbb{C}$ is expressed as $f=\sum_{r} f_{r} \Delta_{r}$, where $f_{r} \in \mathbb{C}$ and $\left\{\Delta_{r}\right\}$ is the dual basis; i.e., $\Delta_{r}\left(L_{s}\right)=\delta_{r, s}$ (and, hence, $\left.f_{r}=f\left(L_{r}\right)\right)$. In the case of $\mathcal{W}$, it follows that $\alpha$ is a 2-coboundary iff there exist constants $\left\{f_{r}\right\}$ such that $\alpha\left(L_{r}, L_{s}\right)=(r-s) \cdot f_{r+s}$; or, equivalently, there exists a function $\bar{f}: \mathbb{Z} \rightarrow \mathbb{C}$ such that

$$
\alpha\left(L_{r}, L_{s}\right)=(r-s) \bar{f}(r+s) .
$$

Observe that the isomorphism class of $\Psi$ is a multiple of Vir and it suffices to compute the quotient of $\Psi\left(\rho\left(L_{r}\right), \rho\left(L_{-r}\right)\right)$ by $\frac{r^{3}-r}{12}$, where $\Psi$ is given by equation (2.17). We do not care about the remainder, since it will take the form of a 2-coboundary. 
Note that the isomorphism class of $\Psi$ does not depend on the conjugacy class of $\rho$, so that it can be assumed that $V=\mathbb{C}((z))$ and, further, $h(z)$ and $c$ are invariant under conjugation (see Section 2.2). Thus, given $h(z), c, b(z)$ and $\rho$ in the form of Theorem 2.1, one has that $\Psi\left(\rho\left(L_{r}\right), \rho\left(L_{-r}\right)\right)$ is the residue at $z=0$ of the following expression

$$
\begin{aligned}
& \frac{1}{3 !}\left(\partial_{z}^{2}\left(\frac{-h(z)^{r+1}}{h^{\prime}(z)}\right) \partial_{z}\left(\frac{-h(z)^{-r+1}}{h^{\prime}(z)}\right)\right) \\
& \quad+\frac{1}{2 !}\left(\partial_{z}\left(\frac{-h(z)^{r+1}}{h^{\prime}(z)}\right) \partial_{z}\left((1-r) \operatorname{ch}(z)^{-r}+\frac{h(z)^{-r+1}}{h^{\prime}(z)} b(z)\right)\right) \\
& \quad+\frac{1}{2 !}\left(\partial_{z}^{2}\left(-(r+1) \operatorname{ch}(z)^{r}+\frac{h(z)^{r+1}}{h^{\prime}(z)} b(z)\right)\left(\frac{-h(z)^{-r+1}}{h^{\prime}(z)}\right)\right) \\
& \quad+\left(\partial_{z}\left(-(r+1) \operatorname{ch}(z)^{r}+\frac{h(z)^{r+1}}{h^{\prime}(z)} b(z)\right)\left((1-r) \operatorname{ch}(z)^{-r}+\frac{h(z)^{-r+1}}{h^{\prime}(z)} b(z)\right)\right) .
\end{aligned}
$$

Expanding this expression and dividing by $\frac{r^{3}-r}{12}$, one obtains the following quotient

$$
-2\left(1-6 c+6 c^{2}\right) \frac{h^{\prime}(z)}{h(z)}
$$

whose residue is equal to

$$
-2\left(1-6 c+6 c^{2}\right) \mathfrak{v}(h(z))
$$

and the result follows.

Remark 2.15. Note that when $c$ is an integer, the central extension admits an alternative geometric construction, namely, the one induced by the action of $\mathbb{C}((z))$ on $\operatorname{Gr}\left(\mathbb{C}((z))(\mathrm{d} z)^{\otimes c}\right)$ (i.e., on the space of $c$-densities). This approach was used in [34] to offer a geometric formalism of the bosonic string. It is worth pointing out that the actions attached to the data $(h(z), c, b(z))$ and to $(h(z), 1-c, b(z))$ are isomorphic.

Remark 2.16. Let $\widehat{\mathcal{D}}$ (a.k.a. $\mathcal{W}_{1+\infty}$ ) denote the unique non-trivial central extension of the Lie algebra of differential operators on the circle. Owing to its presence in several topics, the authors of [16] carried out an in-depth study of its representations with the help of the theory of vertex operator algebras (voa). In this context, they consider two 1-parameter families of Virasoro algebras (see [16, equation (1.7)]) $\left\{L_{k}^{+}(\beta) \mid k \in \mathbb{Z}\right\}$ and $\left\{L_{k}^{-}(\beta) \mid k \in \mathbb{Z}\right\}$, where

$$
\begin{aligned}
& \left\{L_{k}^{+}(\beta)=-z^{k+1} \partial_{z}-\beta(k+1) z^{k} \mid k \geq-1\right\}, \\
& \left\{L_{k}^{-}(\beta)=-z^{k+1} \partial_{z}-(k+\beta(-k+1)) z^{k} \mid k \geq-1\right\},
\end{aligned}
$$

which eventually play a relevant role in their results on conformal voa (see [16, Theorem 3.1.]). Let us check that, for each $\beta$, these Virasoro algebras fit in our approach. For the case $\left\{L_{k}^{+}(\beta)\right\}$ the system is

$$
-\frac{h(z)^{i+1}}{h^{\prime}(z)}=-z^{i+1}, \quad-(i+1) \operatorname{ch}(z)^{i}+\frac{b(z)}{h^{\prime}(z)} h(z)^{i+1}=-\beta(i+1) z^{i}
$$

and it yields $h(z)=z, c=\beta$ and $b(z)=0$. They show that the central charge, $\mathbf{c}(\beta)$, satisfies $\mathbf{c}(\beta)=-2\left(6 \beta^{2}-6 \beta+1\right) \mathbf{c}(1)$, and indeed this agrees with Theorem 2.14. For the second family, $\left\{L_{k}^{-}(\beta)\right\}$, one obtains $h(z)=z, c=1-\beta$ and $b(z)=(1-2 \beta) z^{-1}$. 


\section{$3 \quad \mathrm{KdV}$ and the string equation}

\subsection{Stabilizer}

Following the notation of Section 2.1, let $(V, \rho)$ be an action of $\mathcal{W}^{+}$.

Let $U \subset V$ denote a $\mathbb{C}$-vector subspace and let $A_{U}$ denote its stabilizer; that is

$$
A_{U}:=\operatorname{Stab}(U)=\{f \in \mathbb{C}((z)) \mid f U \subseteq U\} .
$$

We say that $U$ is $L_{-1}$-stable or stable under the action of $L_{-1}$ when $\rho\left(L_{-1}\right) U \subseteq U$. Similarly, we say that $U$ is $\mathcal{W}^{+}$-stable when $\rho(L) U \subseteq U$ for all $L \in \mathcal{W}^{+}$; or, what is tantamount to this, $\rho\left(L_{k}\right) U \subseteq U$ for all $k \geq-1$.

Let us fix the following notation. Let $V_{+} \subseteq V$ denote a $\mathbb{C}[[z]]$-submodule of $V$ and, as above, let $\mathfrak{v}$ be the valuation defined by $z$.

Theorem 3.1. Let $(V, \rho)$ be an action of $\mathcal{W}^{+}$and let $h(z)$ be given as in Theorem 2.1. Let $U$ be a subspace of $V$ such that $U \neq(0), U \cap V_{+}$is finite-dimensional and $\mathfrak{v}(h(z))<0$.

If $U$ is $L_{-1}$-stable and $A_{U} \neq \mathbb{C}$, then $U$ is $\mathcal{W}^{+}$-stable and $A_{U}=\mathbb{C}[h(z)]$.

Proof. First, let us show that $\mathfrak{v}(f(z))<0$ for each $f(z) \in A_{U}$ non-constant. Indeed, if $\mathfrak{v}(f(z))>0$ then $U \cap V_{+}$cannot be finite-dimensional since $U \neq(0)$. On the other hand, if $\mathfrak{v}(f(z))=0$, then $\bar{f}(z):=f(z)-f(0)$ belongs to $A_{U}$ and $\mathfrak{v}(\bar{f}(z))>0$, which again contradicts the hypotheses. Thus, it must hold that $\mathfrak{v}(f(z))<0$.

We shall now prove that $A_{U} \subseteq \mathbb{C}[h(z)]$. From the previous paragraph, let us take $f(z) \in A_{U} \backslash$ $\mathbb{C}[h(z)]$ such that $\mathfrak{v}(f(z))$ attains the value $\max \left\{\mathfrak{v}(f(z)) \mid f(z) \in A_{U} \backslash \mathbb{C}[h(z)]\right\}$. Since $U$ is stable under $L_{-1}$ and under the multiplication by $f(z)$, it follows that $\left[f(z), \rho\left(L_{-1}\right)\right]=\frac{f^{\prime}(z)}{h^{\prime}(z)} \in A_{U}$. Note

that $\mathfrak{v}\left(\frac{f^{\prime}(z)}{h^{\prime}(z)}\right)=\mathfrak{v}(f(z))-\mathfrak{v}(h(z))>\mathfrak{v}(f(z))$, since $\mathfrak{v}(h(z))$ is negative and $\mathfrak{v}(f(z)) \neq 0$. Bearing in mind that $f(z)$ is such that $\mathfrak{v}(f(z))$ is maximal among those elements of $A_{U} \backslash \mathbb{C}[h(z)]$, we have that

$$
\frac{f^{\prime}(z)}{h^{\prime}(z)} \in \mathbb{C}[h(z)]
$$

and, thus, $f(z) \in \mathbb{C}[h(z)]$. That is, $A_{U} \subseteq \mathbb{C}[h(z)]$.

Let us see that $A_{U}=\mathbb{C}[h(z)]$. Since $A_{U} \neq \mathbb{C}$, let $p(x)$ be a non-constant polynomial of minimal degree such that $p(h(z)) \in A_{U}$. Similar to the above, one has that $\left[p(h(z)), \rho\left(L_{-1}\right)\right]=$ $p^{\prime}(h(z)) \in A_{U}$ and, thus, $p^{\prime}(x)$ must be constant and $p(x)$ is of the form $a x+b$. Therefore, $\mathbb{C}[h(z)]=\mathbb{C}[p(h(z))] \subseteq A_{U} \subseteq \mathbb{C}[h(z)]$.

It remains to show that, in the case $A_{U}=\mathbb{C}[h(z)], U$ is $\mathcal{W}^{+}$-stable. This follows from the fact that $h(z) U \subseteq U$ and from the relation $\rho\left(L_{i}\right)=h(z)^{i}\left(h(z) \rho\left(L_{-1}\right)-(i+1) c\right)$ for all $i \geq 0$.

Remark 3.2. The hypothesis $\mathfrak{v}(h(z))<0$ is satisfied in most relevant cases. For instance, if $U$ is $\mathcal{W}^{+}$-stable and $U \cap V_{+}$is finite-dimensional, then $\mathfrak{v}(h(z)) \leq 0$. Indeed, if $\mathfrak{v}(h(z))>0$, then the operator $h(z)^{i}\left(h(z) \rho\left(L_{-1}\right)-(i+1) c\right)$ would raise the order for all $i \gg 0$ and, since $U \neq(0)$, $U \cap V_{+}$could not be of finite dimension.

Remark 3.3. This result clearly unveils why the string equation together with the KdV hierarchy (i.e., $L_{-1}$-stability and $h(z)=z^{-2}$ ) imply the Virasoro constraints (i.e., $\mathcal{W}^{+}$-stability). On the other hand, the existence of points in the Grassmannian with trivial stabilizer that are $L_{-1}$-stable but not $\mathcal{W}^{+}$-stable is known [31, Section 8].

Given an action $(V, \rho)$, let us introduce the first-order stabilizer of a subspace $U \subset V$ as

$$
A_{U}^{1}:=\left\{D \in \mathcal{D}_{\mathbb{C}((z)) / \mathbb{C}}^{1}(V, V) \mid D(U) \subseteq U\right\}
$$


Then, for a $\mathcal{W}^{+}$-stable subspace $U \subset V$ such that $A_{U}=\mathbb{C}[h(z)]$, there is a canonical exact sequence of Lie algebras

$$
0 \longrightarrow A_{U} \longrightarrow A_{U}^{1} \longrightarrow \operatorname{Der}_{\mathbb{C}}\left(A_{U}\right) \longrightarrow 0
$$

and $\rho$ induces a splitting of it. To prove this, one uses the sequence

$$
0 \rightarrow \operatorname{End}_{\mathbb{C}((z))} V \rightarrow \mathcal{D}_{\mathbb{C}((z)) / \mathbb{C}}^{1}(V, V) \rightarrow \operatorname{Der}_{\mathbb{C}}(\mathbb{C}((z))) \rightarrow 0,
$$

together with [18, Remark 3.9] and the explicit expressions of the operators $\rho\left(L_{i}\right)$ computed in Theorem 2.1.

Remark 3.4. Note that if $U$ is $L$-stable for $L \in \mathcal{D}_{\mathbb{C}((z)) / \mathbb{C}}^{1}(V, V)$, it follows that $L \in A_{U} \oplus$ $\operatorname{Im}(\rho)$. Further, given an action $(\mathbb{C}((z)), \rho)$ and a $\mathcal{W}^{+}$-stable point $U \in \operatorname{Gr}(V)$, then $A_{U}^{1}=$ $\mathbb{C}[h(z)] \otimes_{\mathbb{C}}\left\langle 1, \rho\left(L_{-1}\right)\right\rangle$ as a Lie subalgebra of $\mathcal{D}_{\mathbb{C}((z)) / \mathbb{C}}^{1}(V, V)$. It is worth mentioning the paper [1, Section 2.1] where the authors study subspaces of the Sato Grassmannian, which are stable by the multiplication by a power of $z$ as well as by the action of a first-order differential operator. Then, they investigate the matrix integral representation of the corresponding $\tau$-function.

Remark 3.5. Recall from Remark 2.12 that the groups $\mathbb{C}((z))^{*}$ and $\operatorname{SGl}(V)$ act canonically on the Sato Grassmannian. For $U$ a point of $\operatorname{Gr}(V)$, it is well known that the stabilizer, $A_{U}$, coincides with the kernel of the differential of the orbit map, $T_{1} \mathbb{C}((z))^{*} \rightarrow T_{U} \operatorname{Gr}(V)$. Similarly, in the case of semilinear automorphisms the orbit map at the tangent spaces reads as follows

$$
\mathcal{D}_{\mathbb{C}((z)) / \mathbb{C}}^{1}(V, V)=T_{1} \operatorname{SGl}(V) \longrightarrow \operatorname{Hom}_{\mathbb{C}}(U, V / U),
$$

such that the first-order stabilizer $A_{U}^{1}$ is its kernel.

Proposition 3.6. Let $(\mathbb{C}((z)), \rho)$ be an action associated with a triple $(h(z), c, b(z))$. Let $U \subset$ $\mathbb{C}((z))$ be a $\mathcal{W}^{+}$-stable subspace such that $A_{U}=\mathbb{C}[h(z)]$. Let $\widetilde{A}_{U}^{1}$ be the central extension of $A_{U}^{1}$ obtained from the exact sequence (2.15). Let $b(z)=\sum_{i} b_{i} z^{i}$.

If $h(z)=z^{-n}, b_{n i-1}=0$ for all $i \geq 1$ and $b_{-1}=\frac{n+1}{2}$, then the exact sequence of Lie algebras

$$
0 \rightarrow \mathbb{C} \mathbf{c} \rightarrow \widetilde{A}_{U}^{1} \rightarrow A_{U}^{1} \rightarrow 0
$$

splits.

Proof. Similarly to the proof of Theorem 2.14, we shall compute $\Psi$, the Gelfand-Fuchs cocycle (equation (2.17)), for a basis of $A_{U}^{1}$. Recall that a basis is given by $\rho\left(L_{k}\right)$ for $k \geq-1$ and by $h(z)^{k}$ for $k \geq 0$.

Recall that

$$
\Psi\left(\rho\left(L_{r}\right), \rho\left(L_{s}\right)\right)=\left(1-6 c+6 c^{2}\right) n \frac{r^{3}-r}{6} \delta_{r,-s},
$$

which vanishes for $r, s \geq-1$.

Secondly, note that $\Psi\left(h(z)^{r}, h(z)^{s}\right)=-r n \delta_{r,-s}$ and that, hence, it vanishes for $r, s \geq 0$.

Finally, a straightforward computation yields

$$
\begin{aligned}
& \Psi\left(\rho\left(L_{r}\right)+(r+1) \operatorname{ch}(z)^{r}, h(z)^{s}\right)=\Psi\left(-\frac{h(z)^{r+1}}{h^{\prime}(z)} \partial_{z}, h(z)^{s}\right)+\Psi\left(\frac{h(z)^{r+1}}{h^{\prime}(z)} b(z), h(z)^{s}\right) \\
& =\frac{1}{2} \operatorname{Res}_{z=0}\left(\partial_{z}\left(-\frac{h(z)^{r+1}}{h^{\prime}(z)}\right) \partial_{z}\left(h(z)^{s}\right)\right) \mathrm{d} z+\operatorname{Res}_{z=0}\left(\partial_{z}\left(\frac{h(z)^{r+1}}{h^{\prime}(z)} b(z)\right) h(z)^{s}\right) \mathrm{d} z \\
& =\frac{1}{2}(n r-1) n s \delta_{r,-s}+n s b_{n(r+s)-1},
\end{aligned}
$$

which vanishes for all $r \geq-1, s \geq 0$, because of the hypotheses on the coefficients $b_{i}$. 
Example 3.7. Let us consider the case where $h(z)=z^{-2}$ and $b_{-1} \in \frac{1}{2} \mathbb{Z} \backslash \mathbb{Z}$. We will see that there exists $s(z) \in \mathbb{C}((z))$ such that the sequence for $\widetilde{A}_{s(z) U}^{1}$ is split. First of all, note that for $\bar{s}(z)=1+\sum_{i>0} s_{i} z^{2 i} \in \mathbb{C}[[z]]^{*}$, it holds that the stabilizer of $\bar{s}(z) U$ is generated by $h(z)^{r}$ for $r \geq 0$ and by $\rho^{\bar{s}(z)}\left(L_{r}\right)$ for $r \geq-1$ where, as in Section $2.2, \rho^{\bar{s}(z)}$ is the conjugation of $\rho$ by the homothety $\bar{s}(z)$. Then, by Section $2.2, \rho^{\bar{s}(z)}$ is associated with data $\left(h(z)=z^{-2}, c, b(z)-\frac{\bar{s}^{\prime}(z)}{\bar{s}(z)}\right)$. Hence, for $\bar{s}(z)$ satisfying $\frac{\bar{s}^{\prime}(z)}{\bar{s}(z)}=\sum_{i \geq 0} b_{2 i+1} z^{2 i+1}$, we may assume that $b_{2 i+1}=0$. The same argument, for the conjugation by the homothety $z^{k}$ with $k=b_{-1}-\frac{3}{2} \in \mathbb{Z}$, shows that we may assume $b_{-1}=\frac{3}{2}$. That is, the desired series $s(z)$ equals $z^{k} \bar{s}(z)$.

Corollary 3.8. Let $U \subset \mathbb{C}((z))$ be a subspace with $A_{U}=\mathbb{C}[h(z)]$ and stable under an action $(\mathbb{C}((z)), \rho)$ defined by a triple $(h(z), c, b(z))$.

There exists a semilinear transformation $g \in \operatorname{SGl}(\mathbb{C}((z)))$ such that $\widetilde{A}_{g(U)}^{1}$, the central extension of $A_{g(U)}^{1}$, is split iff $b_{-1} \in \frac{1}{2} \mathbb{Z} \backslash \mathbb{Z}$.

Proof. The conclusion follows from Theorem 2.9 and Proposition 3.6.

Remark 3.9. The condition on the residue was also obtained, from another point of view, by Schwarz, see [37, equation (29)].

Let us say a word on the relevance of this corollary. Observe that $A_{U}^{1}$ acts on $\mathbb{C}((z))$ while its central extension, $\widetilde{A}_{U}^{1}$, acts naturally on the fermionic Fock space $H^{0}\left(\operatorname{Gr}(V)\right.$, $\left.\operatorname{Det}_{V}^{*}\right) \simeq \Lambda^{\frac{\infty}{2}} V$. Thus, in order to apply the bosonization isomorphism to relate subspaces and $\tau$-functions (as it was explained in the introduction), it is needed that operators (of our algebra) on $\mathbb{C}((z))$ can be lifted to operators (of the central extension) in a compatible way; that is, that the central extension $\widetilde{A}_{U}^{1}$ is split and, thus, the restriction of the bosonization morphism to it is a Lie algebra homomorphism. Further, having in mind that $U$ is invariant under the action of $\widetilde{A}_{U}^{1}$, it follows that each element of $\widetilde{A}_{U}^{1}$ defines an homothety of the stalk of the determinant bundle at $U$, Det ${ }_{U}^{*}$. Thus, in order to define a canonical section of $\pi: \widetilde{A}_{U}^{1} \rightarrow A_{U}^{1}$ one maps an element $a \in A_{U}^{1}$ to the element of $\pi^{-1}(a)$ such that induces the identity at $\operatorname{Det}_{U}^{*}$. We will come again to this point in Section 3.3.

\subsection{Stable subspaces}

In this subsection we aim to construct explicitly a subspace fulfilling our requirements; namely, invariance under the action and under the homothety $z^{-2}$. Because of this fact and of Theorem 3.1, we shall assume, henceforth, that $h(z)=z^{-2}$.

A naive candidate would be the $\mathbb{C}[h(z)]$-module generated by 1 under the action of $\rho\left(L_{-1}\right)$. Nevertheless, we shall need to consider a conjugate of it (Section 2.2). For this, we shall choose a solution of the Airy equation and decompose $b(z)$ in a suitable way. Let us be more precise.

First, we choose $w(z)$, a formal solution of the Airy equation

$$
w^{\prime \prime}(z)+\frac{1}{2} S(h(z)) w(z)=0,
$$

where $S$ denotes the Schwarzian derivative; that is

$$
S(h):=\frac{h^{\prime \prime \prime}(z)}{h^{\prime}(z)}-\frac{3}{2}\left(\frac{h^{\prime \prime}(z)}{h^{\prime}(z)}\right)^{2} .
$$


It is a straightforward check that $w(z)$ satisfies the Airy equation iff $f(z):=\frac{w^{\prime}(z)}{w(z)}$ satisfies the Riccati equation

$$
f(z)^{2}+f^{\prime}(z)+\frac{1}{2} S(h(z))=0 .
$$

Note, in particular, that $h^{\prime}(z)^{-1 / 2}$ satisfies the equation (3.3); or, equivalently, $\mathrm{d} \log \left(h^{\prime}(z)^{-1 / 2}\right)=$ $-\frac{1}{2} \frac{h^{\prime \prime}(z)}{h^{\prime}(z)}$ satisfies equation (3.4).

Recalling from [26, Chapters 6 and 9] the basic properties of the solutions of the Airy and Riccati equations, we know that in our situation the solutions of equation (3.4) are meromorphic; i.e., $\frac{w^{\prime}(z)}{w(z)} \in \mathbb{C}((z))$. Thus, it makes sense to conjugate a given action by $w(z)$ (see Section 2.2 ).

Furthermore, if $w(z)$ and $h^{\prime}(z)^{-\frac{1}{2}}$ are linearly independent, then the fact that they are solutions of the Airy equation implies that the Schwarzian derivative of $w(z) h^{\prime}(z)^{\frac{1}{2}}$ coincide with that of $h(z)$, and they therefore differ by a Möbius transformation; that is

$$
w(z) h^{\prime}(z)^{\frac{1}{2}}=\frac{\alpha h(z)+\beta}{\gamma h(z)+\delta} \quad \text { for some }\left(\begin{array}{ll}
\alpha & \beta \\
\gamma & \delta
\end{array}\right) \in \operatorname{PGL}(2, \mathbb{C}) .
$$

Second, given a first-order differential operator $P$ we search for a formal expression $v(z)$ such that

$$
P^{v}-u(h(z))=-\frac{1}{h^{\prime}(z)} \partial_{z}-\frac{1}{2} \frac{h^{\prime \prime}(z)}{h^{\prime}(z)},
$$

where $u(x) \in \mathbb{C}[x]$ (the reason is that the square of this operator has no term in $\partial_{z}$ ). More concretely, for the case $P=-\frac{1}{h^{\prime}(z)} \partial_{z}+\frac{b(z)}{h^{\prime}(z)}$, we express $b(z)$ w.r.t. the decomposition

$$
\mathbb{C}((z)) \simeq \mathbb{C}[h(z)] h^{\prime}(z) \oplus\left(\mathbb{C}[h(z)]+\mathbb{C}[[z]] z^{-1}\right) \simeq \mathbb{C}\left[z^{-2}\right] z^{-3} \oplus\left(\mathbb{C}\left[z^{-2}\right]+\mathbb{C}[[z]] z^{-1}\right),
$$

since $h(z)=z^{-2}$ and $h^{\prime}(z)=-2 z^{-3}$. That is, let us consider the unique elements $u(h(z)), v(z)$, where $u(x)$ is a polynomial and $v(z)$ is a formal expression with $\frac{v^{\prime}(z)}{v(z)} \in \mathbb{C}[h(z)]+z^{-1} \mathbb{C}[[z]]$, such that

$$
b(z)=u(h(z)) h^{\prime}(z)+\left(\frac{v^{\prime}(z)}{v(z)}-\frac{1}{2} h^{\prime \prime}(z)\right) .
$$

Equivalently, $v(z)$ is defined by the formal expression

$$
v(z):=\exp \int\left(b(z)-u(h(z)) h^{\prime}(z)+\frac{1}{2} h^{\prime \prime}(z)\right) \mathrm{d} z .
$$

Lemma 3.10. Given $P=-\frac{1}{h^{\prime}(z)} \partial_{z}+\frac{b(z)}{h^{\prime}(z)}$, let $w(z), u(h(z)), v(z)$ as above. It then holds that

$$
\left(P^{2}-2 u(h(z)) P+\left(u^{\prime}(h(z))+u(h(z))^{2}\right)\right)(1 \otimes w(z) v(z))=0 .
$$

Proof. Note that the 1.h.s. in the statement is rewritten as

$$
\begin{aligned}
(P-u(h(z)))^{2}(1 \otimes w(z) v(z)) & =\left(\left(P-u(h(z))-\frac{1}{h^{\prime}(z)} \frac{v^{\prime}(z)}{v(z)}\right)^{2}(1 \otimes w(z))\right) v(z) \\
& =\left(\left(-\frac{1}{h^{\prime}(z)} \partial_{z}-\frac{1}{2} \frac{h^{\prime \prime}(z)}{h^{\prime}(z)}\right)^{2}(1 \otimes w(z))\right) v(z) \\
& =\frac{1}{h^{\prime}(z)^{2}}\left(\left(\partial_{z}^{2}+\frac{1}{2} S(h(z))\right)(1 \otimes w(z))\right) v(z) .
\end{aligned}
$$


In order to see that the last expression vanishes, note that

$$
\begin{aligned}
\partial_{z}^{2}(1 \otimes w(z)) & =\partial_{z}\left(\partial_{z}(1 \otimes w(z))\right)=\partial_{z}\left(\left(\partial_{z}+\frac{w^{\prime}(z)}{w(z)}\right)(1) \otimes w(z)\right) \\
& =\partial_{z}\left(\frac{w^{\prime}(z)}{w(z)} \otimes w(z)\right)=\left(\left(\frac{w^{\prime}(z)}{w(z)}\right)^{\prime}+\left(\frac{w^{\prime}(z)}{w(z)}\right)^{2}\right) \otimes w(z) \\
& =-\frac{1}{2} S(h(z)) \otimes w(z),
\end{aligned}
$$

where the last equality comes from the fact that $\frac{w^{\prime}(z)}{w(z)}$ solves the Riccati equation (3.4).

Remark 3.11. In [21] the authors are able to solve the second-order differential equation $\left(\frac{3}{2} \bar{z}+\right.$ $\left.\frac{1}{2 \bar{z}} \partial_{\bar{z}}-\frac{1}{4 \bar{z}^{2}}\right)^{2} \phi(\bar{z})=\bar{z}^{2} \phi(\bar{z})$ (their $\bar{z}$ variable and our $z$ variable are related by $\bar{z}=\left(\frac{1}{3}\right)^{\frac{1}{3}} z^{-1}$ ) by the substitution $\phi(\bar{z})=\bar{z}^{1 / 2} \exp \left(\frac{2}{3} \bar{z}^{-3}\right) \psi(\bar{z})$ where $\psi(\bar{z})$ is a solution of the Airy equation. However, this makes sense since they show that $\phi(\bar{z})$ has an asymptotic expansion in $\mathbb{C}\left[\left[\bar{z}^{-1}\right]\right]$. Observe that the previous Lemma can be thought of as an abstract formalization of this substitution.

Theorem 3.12 (existence). Let $w(z)$ be a solution of the Airy equation (3.3) linearly independent with $h^{\prime}(z)^{-\frac{1}{2}}$. Let $(\mathbb{C}((z)), \rho)$ be defined by $\left(h(z)=z^{-2}, c, b(z)\right)$ and let $v(z)$ be a formal function such that equation (3.6) is fulfilled. Let $V^{w v}$ be the $\mathbb{C}((z))$-vector space $\mathbb{C}((z)) \otimes w(z) v(z)$ with the conjugated action $\rho^{w v}$.

It then holds that the $\mathbb{C}$-vector subspace of $V^{\text {wv }}$

$$
\mathcal{U}(w):=\left\langle 1 \otimes w(z) v(z), \rho^{w v}\left(L_{-1}\right)(1 \otimes w(z) v(z))\right\rangle \otimes_{\mathbb{C}} \mathbb{C}[h(z)]
$$

is $\mathcal{W}^{+}$-stable, it is a $\mathbb{C}[h(z)]$-module of rank 2 and it belongs to $\operatorname{Gr}\left(V^{w v}\right)$.

Proof. Let us denote $P:=\rho^{w v}\left(L_{-1}\right)$. Lemma 3.10 implies that $\mathcal{U}(w)$ is a $P$-stable $\mathbb{C}[h(z)]$ module. The $\mathcal{W}^{+}$-stability follows from those facts and from the following relations

$$
\begin{aligned}
& \rho^{w v}\left(L_{i}\right)=h(z)^{i}\left(h(z) \rho^{w v}\left(L_{-1}\right)-(i+1) c\right), \\
& \rho^{w v}\left(L_{-1}\right)(p(h(z)) \otimes v(z))=p(h(z)) \rho^{w v}\left(L_{-1}\right)(1 \otimes v(z))-p^{\prime}(h(z)) \otimes v(z) .
\end{aligned}
$$

Let us prove that $P(1 \otimes w(z) v(z)) \notin \mathcal{U}(w) \otimes_{\mathbb{C}[h(z)]} \mathbb{C}\left(\left(h(z)^{-1}\right)\right)$. Observe that

$$
\frac{1}{h^{\prime}(z)} \frac{w^{\prime}(z)}{w(z)}=\frac{1}{h^{\prime}(z)} \mathrm{d} \log \left(w(z) h^{\prime}(z)^{\frac{1}{2}}\right)-\frac{1}{2} \frac{h^{\prime \prime}(z)}{h^{\prime}(z)^{2}} \in \mathbb{C}\left[\left[z^{2}\right]\right]
$$

by equation (3.5) (recall that $h(z)=z^{-2}$ ). Computing how $P$ acts, we have

$$
P(f(z) \otimes w(z) v(z))=\left(-\frac{1}{h^{\prime}(z)} \partial_{z}+u(h(z))-\frac{1}{2} \frac{h^{\prime \prime}(z)}{h^{\prime}(z)}-\frac{1}{h^{\prime}(z)} \frac{w^{\prime}(z)}{w(z)}\right)(f(z)) \otimes w(z) v(z)
$$

and note that the term $\frac{1}{2} \frac{h^{\prime \prime}(z)}{h^{\prime}(z)}$ on the r.h.s. shifts the order by an odd number while all the other terms shift it by an even number. Hence, $\mathcal{U}(w)$ is a free $\mathbb{C}[h(z)]$-module of rank 2 .

Finally, in order to prove that $\mathcal{U}(w)$ lies in the Sato Grassmannian, where we are considering $V_{+}^{w v}:=\mathbb{C}[[z]] \otimes w(z) v(z)$, one has to show the following two conditions; namely,

$$
\begin{aligned}
\operatorname{dim}_{\mathbb{C}}(\mathbb{C}[[z]] \otimes w(z) v(z) \cap \mathcal{U}(w))<\infty, \\
\operatorname{dim}_{\mathbb{C}} \mathbb{C}((z)) \otimes w(z) v(z) /(\mathbb{C}[[z]] \otimes w(z) v(z)+\mathcal{U}(w))<\infty
\end{aligned}
$$

Bearing in mind that $u(h(z))-\frac{1}{2} \frac{h^{\prime \prime}(z)}{h^{\prime}(z)}-\frac{1}{h^{\prime}(z)} \frac{w^{\prime}(z)}{w(z)}$ does not belong to $\mathbb{C}\left(\left(z^{2}\right)\right)$, both conditions follow easily from the previous claims. 
The above constructed subspace depends clearly on the choice of a solution of the Airy equation. The following result studies what this dependence looks like.

Proposition 3.13. Let $(\mathbb{C}((z)), \rho)$ be an action of $\mathcal{W}^{+}$defined by the data $\left\{h(z)=z^{-2}, c, b(z)\right\}$. Let $w_{1}(z), w_{2}(z)$ be two solutions of (3.3).

Then, up to $\mathbb{C}^{*}$, there is a unique isomorphism of $\mathbb{C}((z))$-vector spaces $V^{w_{1}} \stackrel{\sim}{\rightarrow} V^{w_{2}}$ which is compatible w.r.t. the actions of the conjugated actions $\rho^{w_{1}}$ and $\rho^{w_{2}}$.

Proof. We begin by constructing one isomorphism; we shall then prove the uniqueness.

Let us consider $\left(V^{w_{i}}, \rho^{w_{i}}\right)$ as the conjugated action by $w_{i}(z)$ (for $i=1,2$ ); that is, the $\mathbb{C}((z))$-vector space $V^{w_{i}}$ is given by $\mathbb{C}((z)) \otimes_{\mathbb{C}} \mathbb{C} w_{i}(z)$ and $\rho^{w_{i}}$ by equation (2.14).

From [26, Chapter 6], we know that the fact that $w_{1}, w_{2}$ solve (3.3) yields

$$
S\left(\frac{w_{1}(z)}{w_{2}(z)}\right)=S(h)
$$

and that, therefore, there exists $\left(\begin{array}{ll}\alpha & \beta \\ \gamma & \delta\end{array}\right) \in \operatorname{PGL}(2, \mathbb{C})$ such that

$$
\frac{w_{1}(z)}{w_{2}(z)}=\frac{\alpha h(z)+\beta}{\gamma h(z)+\delta}
$$

Let us now check that the $\mathbb{C}((z))$-linear map

$$
\begin{aligned}
& \mathbb{C}((z)) \otimes_{\mathbb{C}} \mathbb{C} w_{1}(z) \longrightarrow \mathbb{C}((z)) \otimes_{\mathbb{C}} \mathbb{C} w_{2}(z), \\
& 1 \otimes w_{1}(z) \mapsto \frac{\alpha h(z)+\beta}{\gamma h(z)+\delta} \otimes w_{2}(z)
\end{aligned}
$$

gives rise to an isomorphism that is compatible with the actions of $\rho^{w_{1}}$ on the l.h.s. and of $\rho^{w_{2}}$ on the r.h.s.; that is, one has to show that

$$
\left(\frac{\alpha h(z)+\beta}{\gamma h(z)+\delta}\right) \rho^{w_{1}}\left(L_{k}\right)\left(f(z) \otimes w_{1}(z)\right)=\rho^{w_{2}}\left(L_{k}\right)\left(\left(\frac{\alpha h(z)+\beta}{\gamma h(z)+\delta}\right) f(z) \otimes w_{2}(z)\right) .
$$

We shall only prove the case $k=-1, f(z)=1$, since the general case goes along the same lines.

First, taking logarithms and derivatives in equation (3.7), we obtain

$$
\frac{w_{1}^{\prime}(z)}{w_{1}(z)}=\frac{w_{2}^{\prime}(z)}{w_{2}(z)}+\left(\frac{\alpha h(z)+\beta}{\gamma h(z)+\delta}\right)^{-1} \partial_{z}\left(\frac{\alpha h(z)+\beta}{\gamma h(z)+\delta}\right) .
$$

On the one hand, one computes the image of

$$
\rho^{w_{1}}\left(L_{-1}\right)\left(1 \otimes w_{1}(z)\right)=\left(-\frac{1}{h^{\prime}(z)} \frac{w_{1}^{\prime}(z)}{w_{1}(z)}+\frac{b(z)}{h^{\prime}(z)}\right) \otimes w_{1}(z)
$$

by the map (3.8) and one obtains

$$
\begin{aligned}
& \frac{\alpha h(z)+\beta}{\gamma h(z)+\delta}\left(-\frac{1}{h^{\prime}(z)} \frac{w_{1}^{\prime}(z)}{w_{1}(z)}+\frac{b(z)}{h^{\prime}(z)}\right) \otimes w_{2}(z) \\
& \quad=-\frac{1}{h^{\prime}(z)}\left(\frac{\alpha h(z)+\beta}{\gamma h(z)+\delta} \frac{w_{2}^{\prime}(z)}{w_{2}(z)}+\partial_{z}\left(\frac{\alpha h(z)+\beta}{\gamma h(z)+\delta}\right)-\frac{\alpha h(z)+\beta}{\gamma h(z)+\delta} b(z)\right) \otimes w_{2}(z),
\end{aligned}
$$

where we have used the identity (3.9). 
On the other hand, one has

$$
\begin{aligned}
& \rho^{w_{2}\left(L_{-1}\right)}\left(\frac{\alpha h(z)+\beta}{\gamma h(z)+\delta} \otimes w_{2}(z)\right) \\
& \quad=\left(\frac{\alpha h(z)+\beta}{\gamma h(z)+\delta}\right)\left(-\frac{1}{h^{\prime}(z)} \frac{w_{2}^{\prime}(z)}{w_{2}(z)}+\frac{b(z)}{h^{\prime}(z)}\right) \otimes w_{2}(z)-\frac{1}{h^{\prime}(z)} \partial_{z}\left(\frac{\alpha h(z)+\beta}{\gamma h(z)+\delta}\right) \otimes w_{2}(z)
\end{aligned}
$$

and, since this expression coincides with equation (3.10), it follows that (3.8) is an isomorphism compatible with the actions.

Let us denote by $\phi$ the isomorphism (3.8) and let $\psi: V^{w_{1}} \rightarrow V^{w_{2}}$ be another isomorphism compatible with the actions. The statement will be proved if we can show that $\phi \circ \psi^{-1}$ belongs to $\mathbb{C}^{*}$.

Let $f(z)$ be defined by $\psi\left(1 \otimes w_{1}(z)\right)=f(z) \otimes w_{2}(z)$. Thus

$$
\left(\phi \circ \psi^{-1}\right)\left(1 \otimes w_{2}(z)\right)=f(z)^{-1} \frac{\alpha h(z)+\beta}{\gamma h(z)+\delta} \otimes w_{2}(z)
$$

is a $\mathbb{C}((z))$-linear automorphism of $V^{w_{2}}$ that is compatible with the action of $\rho^{w_{2}}$; that is, $\left(\phi \circ \psi^{-1}\right) \circ \rho^{w_{2}}=\rho^{w_{2}} \circ\left(\phi \circ \psi^{-1}\right)$ and, bearing in mind Remark 2.5, it follows that

$$
\frac{1}{h^{\prime}(z)} \partial_{z}\left(f(z)^{-1} \frac{\alpha h(z)+\beta}{\gamma h(z)+\delta}\right)=0
$$

and, hence, $f(z)=\lambda \frac{\alpha h(z)+\beta}{\gamma h(z)+\delta}$ for $\lambda \in \mathbb{C}^{*}$ and the statement follows.

Remark 3.14. Let $(V, \rho)$ be an action of $\mathcal{W}^{+}$under the conditions of Theorem 3.12. Let us choose $\gamma \in \mathbb{C}$. Bearing in mind Theorem 2.1, we consider the action defined by

$$
\rho_{\gamma}\left(L_{k}\right):=\rho\left(L_{k}\right)+(k+1) h(z)^{k} \gamma \quad \forall k \geq-1
$$

and note that $\mathcal{U}(w)$ is stable under the action of $\mathcal{W}^{+}$via $\rho_{\gamma}$ for all $\gamma$; that is, there exists a 1-parameter family of actions preserving the same subspace $\mathcal{U}(w)$.

Proposition 3.15. Let $\left(V, \rho_{i}\right)(i=1,2)$ be actions of $\mathcal{W}^{+}$defined by the data $h_{i}(z), c_{i}, b_{i}(z)$ as in Theorem 2.1 and let us assume that $\mathfrak{v}\left(h_{i}(z)\right)<0$.

If there exists a subspace $U \in \operatorname{Gr}(V)$ which is $\rho_{i}\left(\mathcal{W}^{+}\right)$-stable for $i=1,2$, then there exist $\alpha \in \mathbb{C}^{*}, \beta \in \mathbb{C}$ such that:

(i) $h_{1}(z)=\alpha h_{2}(z)+\beta$;

(ii) $\rho_{1}\left(L_{-1}\right)-\alpha \rho_{2}\left(L_{-1}\right)=\frac{1}{h_{1}^{\prime}(z)}\left(b_{1}(z)-b_{2}(z)\right) \in A_{U}$.

Conversely, if there exist $\alpha \in \mathbb{C}^{*}, \beta \in \mathbb{C}$ such that $(i)$ and (ii) hold, then a subspace $U \in \operatorname{Gr}(V)$ is $\rho_{1}\left(\mathcal{W}^{+}\right)$-stable if and only if it is $\rho_{2}\left(\mathcal{W}^{+}\right)$-stable.

Proof. First, note that Theorem 3.1 implies that

$$
\mathbb{C}\left[h_{1}(z)\right]=A_{U}=\mathbb{C}\left[h_{2}(z)\right],
$$

such that there exist $\alpha \in \mathbb{C}^{*}, \beta \in \mathbb{C}$ satisfying the first item.

Observe that $\sigma\left(\rho_{1}\left(L_{-1}\right)\right)=\sigma\left(\alpha \rho_{2}\left(L_{-1}\right)\right)=-\frac{1}{h_{1}^{\prime}(z)}$ and, thus

$$
\rho_{1}\left(L_{-1}\right)-\alpha \rho_{2}\left(L_{-1}\right) \in \mathbb{C}((z)) .
$$

Since $U$ is stable under the $\rho_{1}\left(L_{-1}\right)-\alpha \rho_{2}\left(L_{-1}\right)$, the element $\rho_{1}\left(L_{-1}\right)-\alpha \rho_{2}\left(L_{-1}\right)$ must belong to the stabilizer, $A_{U}$.

It follows from Theorem 3.1 that

$$
\rho_{1}\left(L_{-1}\right)-\alpha \rho_{2}\left(L_{-1}\right) \in \mathbb{C}\left[h_{1}(z)\right]
$$

and the first part of the statement follows.

The converse follows from Theorems 3.1 and 2.1. 


\section{$3.3 \tau$-functions}

It is common to work with the second derivatives of the logarithm of the $\tau$-function instead of with the $\tau$-function itself. Indeed, if one looks at the KP hierarchy expressed as Hirota bilinear equations, one realizes that multiplication by the exponential of a linear function preserves the set of solutions of the hierarchy. Accordingly the hierarchy can be equivalently stated for the second derivatives of the logarithm of the $\tau$-function. Also, recall [39, Lemma 3.8], which states that, for $U \in \operatorname{Gr}(V)$, the function $\exp \left(\sum_{i} a_{i} t_{i}\right) \tau_{U}(t)$ is the $\tau$-function of the point $\exp \left(-\sum_{i} a_{i} \frac{z^{i}}{i}\right) U \in \operatorname{Gr}(V)$; that is, $\partial_{t_{i}} \partial_{t_{j}} \log \tau_{U}(t)$ does not vary on the orbit of $U \in \operatorname{Gr}(V)$ under the action of $\mathbb{C}[[z]]^{*}$ by homotheties. Thus, our following results will be focused on the study of $\partial_{t_{i}} \partial_{t_{j}} \log \tau_{U}(t)$ instead of on $\tau_{U}(t)$. Finally, it is worth noticing that these expressions also appear in other topics such as the study of monodromy-preserving deformations, Frobenius manifolds, etc.

As it was mentioned in the introduction, a key point in our approach relies on the equivalence of conditions on subspaces (e.g., $\tilde{L} U \subseteq U$ ) and on their corresponding $\tau$-functions (i.e., $\left.\bar{L} \tau_{U}(t)=0\right)$ where the operators are related through the bosonization isomorphism. Nevertheless, now we are dealing with a set of operators that generate a Lie algebra; namely, $A_{U}^{1}$, rather than just one operator. On the other hand, as it was explained in Section 2.3, the bosonization isomorphism involves the central extension $\widetilde{A}_{U}^{1}$. Thus, in order to make of the bosonization isomorphism a morphism of Lie algebras, we demand the central extension $\widetilde{A}_{U}^{1}$ to be split.

Summing up, from now on, we shall assume that $h(z)=z^{-2}$ and $b_{-1} \in \frac{1}{2} \mathbb{Z} \backslash \mathbb{Z}$ (see Proposition 3.6 and Corollary 3.8).

For an action $(\mathbb{C}((z)), \rho)$ let $\bar{\rho}$ denote the action induced on $\mathbb{C}\left[\left[t_{1}, t_{2}, \ldots\right]\right]$ through the bosonization isomorphism; that is, $\bar{\rho}\left(L_{k}\right):=B \circ \rho\left(L_{k}\right) \circ B^{-1}$ as a differential operator on $\mathbb{C}\left[\left[t_{1}, \ldots\right]\right]$. Recall that if $\rho$ corresponds to a triple $\left(h(z)=z^{-2}, c, b(z)\right)$ (KdV case), one usually deals only with functions of $t$ variables with odd subindices and, therefore, if no confusion arises, the composition

$$
\mathbb{C}\left[\left[t_{1}, t_{3}, \ldots\right]\right] \hookrightarrow \mathbb{C}\left[\left[t_{1}, t_{2}, \ldots\right]\right] \stackrel{\bar{\rho}\left(L_{k}\right)}{\longrightarrow} \mathbb{C}\left[\left[t_{1}, t_{2}, \ldots\right]\right] /\left(t_{2}, t_{4}, \ldots\right) \stackrel{\sim}{\longrightarrow} \mathbb{C}\left[\left[t_{1}, t_{3}, \ldots\right]\right]
$$

will be denoted by $\bar{\rho}\left(L_{k}\right)$ too. Hence, $\bar{\rho}$ can be thought of as acting on $\mathbb{C}\left[\left[t_{1}, t_{3}, \ldots\right]\right]$.

Theorem 3.16 (independence of choices). Let $(\mathbb{C}((z)), \rho)$ be an action of $\mathcal{W}^{+}$such that $h(z)=$ $z^{-2}$ and $b_{-1} \in \frac{1}{2} \mathbb{Z} \backslash \mathbb{Z}$. Let $w_{1}(z), w_{2}(z)$ be solutions of the Airy equation (3.3).

If the hypothesis of Theorem 3.12 holds and $\mathcal{U}\left(w_{i}\right) \in \mathrm{Gr} V^{w_{i} v}$ is the point given by that theorem $(i=1,2)$, then

$$
\partial_{t_{2 i+1}} \partial_{t_{2 j+1}} \log \tau_{\mathcal{U}\left(w_{1}\right)}(t)=\partial_{t_{2 i+1}} \partial_{t_{2 j+1}} \log \tau_{\mathcal{U}\left(w_{2}\right)}(t) \quad \forall i, j \geq 0
$$

and this function is a common solution of the KdV hierarchy and of the Virasoro constraint equations

$$
\bar{\rho}^{w_{i} v}\left(L_{k}\right) \tau_{\mathcal{U}\left(w_{i}\right)}(t)=0 \quad \forall k \geq-1, \quad i=1,2 .
$$

Proof. Note that the isomorphism $V^{w_{1} v} \simeq V^{w_{2} v}$ provided by Proposition 3.13 yields an isomorphism $\operatorname{Gr}\left(V^{w_{1} v}\right) \simeq \operatorname{Gr}\left(V^{w_{2} v}\right)$ which sends $\mathcal{U}\left(w_{1}\right)$ to $\frac{\alpha h(z)+\beta}{\gamma h(z)+\delta} \mathcal{U}\left(w_{2}\right)$, where

$$
\frac{w_{1}(z)}{w_{2}(z)}=\frac{\alpha h(z)+\beta}{\gamma h(z)+\delta}
$$

(see the proof of the proposition).

To begin with, let us assume that $\mathfrak{v}\left(\frac{w_{1}(z)}{w_{2}(z)}\right)=0$ and, thus, $\frac{\alpha h(z)+\beta}{\gamma h(z)+\delta}$ can be expanded as a series in $h(z)^{-1}$, say $g\left(h(z)^{-1}\right) \in \mathbb{C}\left[\left[h(z)^{-1}\right]\right]^{*}=\mathbb{C}\left[\left[z^{2}\right]\right]^{*} \subseteq \mathbb{C}[[z]]^{*}$. 
Bearing in mind [39, Lemma 3.8], we know that the $\tau$-function of $g\left(h(z)^{-1}\right) \mathcal{U}\left(w_{2}\right)$ is equal to the $\tau$-function of $\mathcal{U}\left(w_{2}\right)$ up to the exponential of a linear function on the $t$ variables. Hence, the second derivatives of their logarithms do coincide, i.e.,

$$
\partial_{t_{2 i+1}} \partial_{t_{2 j+1}} \log \tau_{\mathcal{U}\left(w_{2}\right)}(t)=\partial_{t_{2 i+1}} \partial_{t_{2 j+1}} \log \tau_{g \mathcal{U}\left(w_{2}\right)}(t) .
$$

On the other hand, since $\mathfrak{v}\left(\frac{w_{1}(z)}{w_{2}(z)}\right)=0$ the isomorphism of the Proposition 3.13 sends $V_{+}^{w_{1} v}:=$ $\mathbb{C}[[z]] \otimes_{\mathbb{C}} \mathbb{C} w_{1}(z) \otimes_{\mathbb{C}} \mathbb{C} v(z)$ to $V_{+}^{w_{2} v}:=\mathbb{C}[[z]] \otimes_{\mathbb{C}} \mathbb{C} w_{2}(z) \otimes_{\mathbb{C}} \mathbb{C} v(z)$ and, hence, it is compatible with the construction of the $\tau$-functions because $\tau$-functions are defined as the determinant of the projection onto $V^{w_{1} v} / V_{+}^{w_{1} v}$, resp. $V^{w_{2} v} / V_{+}^{w_{2} v}$ (see [39] or [3, Definition 5.6]). Therefore, the $\tau$-function of $\mathcal{U}\left(w_{1}\right)$ and that of its image, $g\left(h(z)^{-1}\right) \mathcal{U}\left(w_{2}\right)$, coincide. The claim is proved.

It remains to check the cases where $\mathfrak{v}\left(\frac{w_{1}(z)}{w_{2}(z)}\right) \neq 0$. Observe that there are two possibilities: either it is 2 or -2 . Let us assume that it is 2 ; that is, its expansion lies in $h(z)^{-1} \mathbb{C}\left[\left[h(z)^{-1}\right]\right]^{*}=$ $z^{2} \mathbb{C}[[z]]^{*}$ and the isomorphism induced between the Grassmannians sends the connected component of index $\lambda$ to the connected component of index $\lambda+2$. Recalling that if the $\tau$-function of a point in $\operatorname{Gr}^{\lambda}\left(V^{w_{2} v}\right)$ is the determinant of the projection onto $V^{w_{2} v} / z^{\lambda} V_{+}^{w_{2} v}$, the $\tau$-function of a point in $\mathrm{Gr}^{\lambda+2}\left(V^{w_{2} v}\right)$ is therefore the determinant of the projection onto $V^{w_{2} v} / z^{\lambda+2} V_{+}^{w_{2} v}$. Now, we can proceed as above.

Theorem 3.17 (uniqueness). With $h(z)=z^{-2}$. For $i=1,2$, let $\left(\mathbb{C}((z)), \rho_{i}\right)$ be an action of $\mathcal{W}^{+}$ and let $\tau_{i}(t) \in \mathbb{C}\left[\left[t_{1}, t_{3}, \ldots\right]\right]$ be a $\tau$-function for the $K d V$ hierarchy verifying Virasoro constraints

$$
\bar{\rho}_{i}\left(L_{k}\right)\left(\tau_{i}(t)\right)=0 .
$$

The following three conditions are equivalent:

(i) $\partial_{t_{2 i+1}} \partial_{t_{2 j+1}} \log \tau_{1}(t)=\partial_{t_{2 i+1}} \partial_{t_{2 j+1}} \log \tau_{2}(t)$ for all $i, j \geq 0$;

(ii) $\rho_{1}\left(L_{-1}\right)-\rho_{2}\left(L_{-1}\right) \in \mathbb{C}\left(\left(z^{2}\right)\right)$;

(iii) $\bar{\rho}_{1}=\bar{\rho}_{2}$ on $\mathbb{C}\left[\left[t_{1}, t_{3}, \ldots\right]\right]$.

Proof. First, since $\tau_{i}(t)$ is a $\tau$-function for the KdV hierarchy, the Sato theory implies that it is the $\tau$-function of a subspace $U_{i} \in \operatorname{Gr}(\mathbb{C}((z)))$, such that $z^{-2} U_{i} \subset U_{i}$.

Assuming that item (i) holds, we have that $\tau_{1}(t)$ and $\tau_{2}(t)$ differ by a factor that is the exponential of a linear function in the $t$ variables (with odd subindices). Lemma 3.8 of [39] implies that there are $\alpha_{i} \in \mathbb{C}$ such that $\tau_{1}(t)$ coincides with the $\tau$-function of $f(z) U_{2}$, where $f(z)$ is $\exp \left(-\sum_{i \geq 0} \alpha_{2 i+1} \frac{z^{2 i+1}}{2 i+1}\right)$.

Hence, $\tau_{1}(t)$ is annihilated by $\operatorname{Im} \bar{\rho}_{1}$ and by $\operatorname{Im} \bar{\rho}_{2}^{f}$, with $\rho_{2}^{f}:=f(z) \circ \rho_{2} \circ f(z)^{-1}$. Bearing in mind Proposition 3.15 and the assumption that $h_{1}(z)=h_{2}(z)=h(z)=z^{-2}$, it follows that

$$
\rho_{2}\left(L_{-1}\right)-\rho_{2}^{f}\left(L_{-1}\right) \in \mathbb{C}\left[z^{-2}\right] .
$$

Recalling the explicit expressions (2.1), we have

$$
\begin{aligned}
\rho_{1}\left(L_{-1}\right)-\rho_{2}^{f}\left(L_{-1}\right) & =\frac{1}{h_{1}^{\prime}(z)}\left(b_{1}(z)-\left(b_{2}(z)-\frac{\partial_{z} \exp \left(\sum_{i \geq 0} \alpha_{2 i+1} \frac{z^{2 i+1}}{2 i+1}\right)}{\exp \left(\sum_{i \geq 0} \alpha_{2 i+1} \frac{z^{2 i+1}}{2 i+1}\right)}\right)\right) \\
& =\frac{1}{h^{\prime}(z)}\left(b_{1}(z)-b_{2}(z)+\sum_{i \geq 0} \alpha_{2 i+1} z^{2 i}\right),
\end{aligned}
$$

which lies in $\mathbb{C}[h(z)]=\mathbb{C}\left[z^{-2}\right]$, and item (ii) is proved. 
Recall that the action of $z^{-2 m}$ (resp. $z^{2 m}$ ) for $m>0$ on the fermionic Fock space corresponds to the action of $\partial_{t_{2 m}}\left(\right.$ resp. $2 m t_{2 m}$ ) on the bosonic Fock space, $\mathbb{C}\left[\left[t_{1}, t_{2}, \ldots\right]\right]$ (this correspondence will be discussed in Section 4.1). Hence the operator $\bar{\rho}\left(L_{-1}\right)-\bar{\rho}_{2}\left(L_{-1}\right)$ acts on $\mathbb{C}\left[\left[t_{1}, t_{2}, \ldots\right]\right]$ as a linear combination of $\partial_{t_{2 m}}$ and $2 m t_{2 m}$. Considering their actions on $\mathbb{C}\left[\left[t_{1}, t_{3}, \ldots\right]\right]$ as in $(3.11)$, we conclude item (iii).

Reversing the arguments, the converse implications (iii) $\Longrightarrow$ (ii) $\Longrightarrow$ (i) follow easily.

Remark 3.18. Note that (iii) implies that for all $k \geq-1$ the systems of differential equations $\bar{\rho}_{i}\left(L_{k}\right) f(z)=0$ coincide for $i=1,2$. On the other hand, if one replaces the hypothesis $h_{1}(z)=$ $h_{2}(z)=z^{-2}$ by $h_{1}(z)=\alpha h_{2}(z)+\beta=z^{-2}$ for some $\alpha \in \mathbb{C}^{*}$ and $\beta \in \mathbb{C}$, then (ii) must be replaced by $\mathbb{C}\left(\left(z^{2}\right)\right)+\operatorname{Im} \rho_{1}=\mathbb{C}\left(\left(z^{2}\right)\right)+\operatorname{Im} \rho_{2} ;$ and (iii) by $\operatorname{Im} \bar{\rho}_{1}=\operatorname{Im} \bar{\rho}_{2}$ on $\mathbb{C}\left[\left[t_{1}, t_{3}, \ldots\right]\right]$. These conditions are intimately related to the first-order stabilizer (see Section 3.1).

The moral of this section is that we have been able to define arrow $B$ of diagram (1.1) of the Introduction and study some of its properties. Summarizing, the previous Theorems show that: first, there is an injection

$$
\left\{\begin{array}{c}
\tau(t) \in \mathbb{C}\left[\left[t_{1}, t_{3}, \ldots\right]\right] \\
\text { satisfying } \mathrm{KdV} \text { and string } \\
\text { equation } \bar{\rho}\left(L_{-1}\right) \tau(t)=0
\end{array}\right\} / \sim \underset{\overline{\bar{B}}}{\longrightarrow}\left\{\begin{array}{c}
\rho \in \operatorname{Hom}_{\text {Lie-alg }}\left(\mathcal{W}^{+}, \mathcal{D}^{1}\right) \\
\text { with } \sigma\left(\rho\left(L_{-1}\right)\right)=-\frac{1}{2} z^{3}
\end{array}\right\} / \sim
$$

where $\tau_{1}(t)$ and $\tau_{2}(t)$ are identified when the second derivatives of their logarithms coincide, and $\rho_{1}$ and $\rho_{2}$ are equivalent when $\rho_{1}\left(L_{-1}\right)-\rho_{2}\left(L_{-1}\right) \in \mathbb{C}\left(\left(z^{2}\right)\right)$. And second, that the arrow $\bar{B}$ can be defined on the subset of those $\rho$ such that $b_{-1} \in \frac{1}{2} \mathbb{Z} \backslash \mathbb{Z}$.

\section{Applications}

\subsection{D quantum gravity}

Among the variety of topics in which Virasoro constraints and KdV hierarchy appear together, we have chosen the case of case of $2 \mathrm{D}$ quantum gravity within the framework of conformal field theory. We do not aim to review the literature on CFT exhaustively, but to illustrate how our results may help in the understanding of some mathematical issues of the symmetries of that theory (e.g., the partition function). Thus, our main references for this section will be $[8,9,21,22,25]$. We shall see that the Lie algebras considered by those authors correspond, in the fermionic formulation, to representations of $\mathcal{W}^{+}$on $V=\mathbb{C}((z))$ which fit into the approach offered in Section 2. Further, the data $h(z), c, b(z)$ of Theorem 2.1 will be written down explicitly.

Recall that the bosonization isomorphism, a.k.a. boson-fermion correspondence (e.g., [20, Lecture 5], [22, Section 2]), establishes an $\mathbb{C}$-linear isomorphism $B$ between $\Lambda^{\frac{\infty}{2}} \mathbb{C}((z))$ and $\mathbb{C}\left[\left[t_{1}, t_{2}, \ldots\right]\right]$ that preserves charge and degree. It is well known that $B$ can be explicitly expressed in different flavors such as Schur polynomials, Matrix integrals or Laplace transforms. Therefore, conjugating by $B$, any operator on the bosonic Fock space, $\mathbb{C}\left[\left[t_{1}, t_{2}, \ldots\right]\right]$, can be understood as an operator on the fermionic Fock space, $\Lambda^{\frac{\infty}{2}} \mathbb{C}((z))$. It is worth noticing that if we are given an algebra of operators on the bosonic Fock space that is isomorphic to the Virasoro algebra (or to $\mathcal{W}^{+}$), then the algebra generated by their conjugations w.r.t. $B$ is also isomorphic to the Virasoro algebra (or to $\mathcal{W}^{+}$). As an example, we refer readers to [23, Table 1] where an explicit description on how the same operator acts on both spaces is given. 
In [22, Section 2.2] the authors explain how the bosonic formulation associated with the central charge $\mathbf{c}=1$ and the charge 0 sector corresponds to the action of the fields

$$
\begin{aligned}
& \bar{L}_{-n}=\sum_{m=1}^{\infty}(n+m) t_{n+m} \partial_{t_{m}}+\frac{1}{2} \sum_{m=1}^{n-1} m(n-m) t_{n-m} t_{m}, \quad \bar{L}_{0}=\sum_{m=1}^{\infty} m t_{m} \partial_{t_{m}}, \\
& \bar{L}_{n}=\sum_{m=1}^{\infty} m t_{m} \partial_{t_{n+m}}+\frac{1}{2} \sum_{m=1}^{n-1} \partial_{t_{m}} \partial_{t_{n-m}}, \quad \mathbf{c}=1
\end{aligned}
$$

(where $n \geq 1$ ) in the Fock space $\mathbb{C}\left[\left[t_{1}, t_{2}, \ldots\right]\right]$.

Let us translate this picture into the fermionic formulation. It is well known the explicit correspondence between certain first-order differential operators on $\mathbb{C}((z))$ and certain differential operators on the Fock space (see, for instance, [23, Table 1]; see [17] for an approach based on quantization). Indeed, if $a_{m}$ acts on $\mathbb{C}((z))$ by the homothety $z^{n}$, then it acts on the Fock space by

$$
a_{n}:= \begin{cases}n t_{n} & \text { for } n>0 \\ 0 & \text { for } n=0 \\ \partial_{t_{-n}} & \text { for } n<0\end{cases}
$$

For $n \geq 1$, it holds that the action of the operator $z^{n}\left(z \partial_{z}+\frac{1+n}{2}\right)$ acting on $\mathbb{C}((z))$ corresponds to the following operator on the bosonic Fock space

$$
\begin{aligned}
\frac{1}{2} \sum_{m=-\infty}^{\infty}: a_{m} a_{n-m}: & =\frac{1}{2} \sum_{m=1}^{n-1} a_{m} a_{n-m}+\frac{1}{2} \sum_{m=n+1}^{\infty} a_{m} a_{n-m}+\frac{1}{2} \sum_{m=-1}^{-\infty} a_{n-m} a_{m} \\
& =\frac{1}{2} \sum_{m=1}^{n-1} a_{m} a_{n-m}+\frac{1}{2} \sum_{m=1}^{\infty} a_{n+m} a_{-m}+\frac{1}{2} \sum_{m=1}^{\infty} a_{n+m} a_{-m} \\
& =\frac{1}{2} \sum_{m=1}^{n-1} m(n-m) t_{m} t_{n-m}+\sum_{m=1}^{\infty}(m+n) t_{n+m} \partial_{t_{m}}=\bar{L}_{-n},
\end{aligned}
$$

where : : denotes the normal ordering. Analogously, the action of $z^{-n}\left(z \partial_{z}+\frac{1-n}{2}\right)$ on $\mathbb{C}((z))$ corresponds to the action of

$$
\frac{1}{2} \sum_{m=-\infty}^{\infty}: a_{m} a_{-n-m}:=\bar{L}_{n} .
$$

Hence, the action of $\mathcal{W}^{+}$in $V=\mathbb{C}((z))$ corresponding to $\left\{\bar{L}_{n} \mid n \geq-1\right\}$ is explicitly given by

$$
\rho\left(L_{n}\right):=z^{-n}\left(z \partial_{z}+\frac{1-n}{2}\right) \quad \forall n \geq-1 .
$$

Finally, let us compute $h(z), b(z)$ and $c$ from the equations

$$
-\frac{h(z)^{n+1}}{h^{\prime}(z)}=z^{-n+1}, \quad-(n+1) \operatorname{ch}(z)^{n}+\frac{b(z)}{h^{\prime}(z)} h(z)^{n+1}=\frac{1-n}{2} z^{-n}
$$

and, therefore, $h(z)=z^{-1}, c=\frac{1}{2}$ and $b(z)=-z^{-1}$.

Let us now discuss how the KdV hierarchy shows up. Recall that, for $m=2$ and the above data, Example 2.8 provides a method to obtain another action with $h(z)=z^{-2}$, which corresponds to the $\mathrm{KdV}$. We shall see that this is indeed the case for $2 \mathrm{D}$ gravity. 
Douglas [9] proposed that the non-perturbative partition function of two-dimensional gravity is the square of a $\tau$-function for the $\mathrm{KdV}$ hierarchy that also satisfies the string equation. In [8] the authors discussed further consequences for the case of topological gravity but, again, the constraints arised from the KdV hierarchy and from the string equation. Let us see how. In their paper we find a Lie algebra, that is isomorphic to $\mathcal{W}^{+}$, and that is generated by the following operators (see [8, equation $(3.5)]$ )

$$
\begin{aligned}
& \bar{L}_{-1}^{\prime}=\sum_{i=1}^{\infty}\left(i+\frac{1}{2}\right) q_{i} \partial_{q_{i-1}}+\frac{1}{8} \lambda^{-2} q_{0}^{2}, \quad \bar{L}_{0}^{\prime}=\sum_{i=0}^{\infty}\left(i+\frac{1}{2}\right) q_{i} \partial_{q_{i}}+\frac{1}{16}, \\
& \bar{L}_{n}^{\prime}=\sum_{i=0}^{\infty}\left(i+\frac{1}{2}\right) q_{i} \partial_{q_{i+n}}+\frac{1}{2} \lambda^{2} \sum_{i=1}^{n} \partial_{q_{i-1}} \partial_{q_{n-i}} \quad n \geq 1
\end{aligned}
$$

acting on $\mathbb{Q}\left[q_{0}, q_{1}, \ldots\right]$. Note that this algebra coincides with the one considered by Givental [17, Section 3] up to rescalling. Here, for the sake of simplicity, the constant term $\frac{1}{16}$ has been incorporated to $\bar{L}_{0}^{\prime}$ so that $\left\{\bar{L}_{n}^{\prime} \mid n \geq-1\right\}$ has Lie bracket $\left[\bar{L}_{i}^{\prime}, \bar{L}_{j}^{\prime}\right]=(i-j) \bar{L}_{i+j}^{\prime}$ for $i, j \geq-1$.

Let us explain the relationship between the operators of equation (4.1) and those of equation (4.3). Indeed, if one writes down how the operators of equation (4.1) act on the subspace $\mathbb{C}\left[\left[t_{1}, t_{3}, t_{5}, \ldots\right]\right] \subset \mathbb{C}\left[\left[t_{1}, t_{2}, t_{3}, \ldots\right]\right]$ (since we are dealing with $\tau$-functions for the KdV hierarchy that depend only on $t$ variables with odd subindices), one has

$$
\begin{aligned}
& \bar{L}_{-2 n}=\sum_{m=0}^{\infty}(2(n+m)+1) t_{2(n+m)+1} \partial_{t_{2 m+1}}+\frac{1}{2} \sum_{m=0}^{n-1}(2 m+1)(2(n-m)+1) t_{2(n-m)+1} t_{2 m+1}, \\
& \bar{L}_{0}=\sum_{m=0}^{\infty}(2 m+1) t_{2 m+1} \partial_{t_{2 m+1}}, \\
& \bar{L}_{2 n}=\sum_{m=0}^{\infty}(2 m+1) t_{2 m+1} \partial_{t_{2(n+m)+1}}+\frac{1}{2} \sum_{m=0}^{n-1} \partial_{t_{2 m+1}} \partial_{t_{2(n-m)+1}}, \quad \mathbf{c}=1,
\end{aligned}
$$

where $n \geq 1$. Letting $q_{m}:=\sqrt{2} \lambda t_{2 m+1}$, we observe that $\frac{1}{2} \bar{L}_{2 n}+\delta_{n, 0} \frac{1}{16}$ coincide exactly with the operator $\bar{L}_{n}^{\prime}$ of (4.3). Furthermore, the action of $\mathcal{W}^{+}$on $\mathbb{C}((z))$ corresponding to the approach of [8]; that, to the Lie algebra $\left\{\bar{L}_{n}^{\prime} \mid n \geq-1\right\}$, is

$$
\rho^{\prime}\left(L_{n}\right):=\frac{1}{2} \rho\left(L_{2 n}\right)=\frac{1}{2} z^{-2 n}\left(z \partial_{z}+\frac{1-2 n}{2}\right) \quad \forall n \geq-1,
$$

which is the action defined by the data $h(z)=z^{-2}, c=\frac{1}{2}$ and $b(z)=-\frac{3}{2} z^{-1}$. Note that, as stated above, these data can be obtained by the method of Example 2.8 from $h(z)=z^{-1}, c=\frac{1}{2}$ and $b(z)=-z^{-1}$ (defining the action (4.2)) with $m=2$.

Remark 4.1. Observe that the operators $\left\{\rho^{\prime}\left(L_{n}\right)\right\}$ preserve the decomposition $\mathbb{C}((z))=\mathbb{C}\left(\left(z^{2}\right)\right)$ $\oplus z \mathbb{C}\left(\left(z^{2}\right)\right)$. Indeed, recalling the definitions $J(\bar{z})=\sum_{n=0}^{\infty} q_{n} \bar{z}^{n+1 / 2}[8$, formula $(2.15)]$ and $L_{m}=$ $\bar{z}^{-1 / 2}\left(\bar{z} \frac{\partial}{\partial \bar{z}}\right)^{m} \bar{z}^{-1 / 2}\left[17\right.$, Section 3] we observe that $\bar{z}^{1 / 2}$ plays a relevant role in them (set $\bar{z}=z^{2}$ for a comparison with our setup). It is worth pointing out that both papers deal with $\mathrm{KdV}$ $\tau$-functions.

Remark 4.2. In [23] the author aims to describe the relationship between several equations for Hurwitz numbers and Hodge integrals. There, the first-order differential operators on the Fock space are $\frac{1}{2} z^{-2 n}\left(z \partial_{z}+\frac{1-2 n}{2}\right)$ for $n \geq-1$ which generate a Virasoro algebra [23, Section 6]. From a straightforward computation it follows that this action of $\mathcal{W}^{+}$is defined by $h(z)=z^{-2}$, $c=\frac{1}{2}$ and $b(z)=-\frac{3}{2} z^{-1}$ (applying Theorem 2.1) and it therefore coincides with that of [8]. 
At the time when the previously cited papers were published, Kac and Schwarz [21] wondered about an explicit description of a point in the Sato Grasmannian whose $\tau$-function is the $\tau$ function arising in $2 \mathrm{D}$ gravity (see [8]).

First, let us note that the coordinate they used, $\bar{z}$, and the one we are using are related by $\bar{z}=\left(\frac{1}{3}\right)^{\frac{1}{3}} z^{-1}$. We shall rewrite their statements in terms of $z$ but, for the sake of simplicity, let us start backwards.

The point in the Grassmannian is characterized by the fact that it is the unique point $U$ invariant under multiplication by $z^{-2}$ (which is equivalent to the KdV) and under the action of $A:=\frac{3}{2} \bar{z}+\frac{1}{2} \frac{1}{\bar{z}} \partial_{\bar{z}}-\frac{1}{4} \bar{z}^{-2}=-3^{\frac{2}{3}}\left(-\frac{1}{2} z^{-1}+\frac{1}{2} z^{3} \partial_{z}+\frac{1}{4} z^{2}\right)$ or, equivalently, that $z^{-2} u(z), A u(z) \in U$ for all $u(z) \in U$. It is worth pointing out that $\mathfrak{v}(A u(z))=\mathfrak{v}(u(z))-1$ for all $u(z) \neq 0$ and that

$$
\left[A, z^{-2}\right]=3^{2 / 3} \text {. }
$$

Hence, for a subspace $U \in \operatorname{Gr} \mathbb{C}((z))$ stable under the action of $A$ and $z^{-2}$, let $u_{0}(z) \in U$ be the monic element such that $\mathfrak{v}\left(u_{0}(z)\right)$ attains the maximum of $\{\mathfrak{v}(u(z)) \mid u(z) \in U\}$. It then follows that $u_{0}(z), A u_{0}(z), A^{2} u_{0}(z)$ and $z^{-2} u_{0}(z)$ must be linearly dependent over $\mathbb{C}$. After some computations the authors show that the linear dependence implies that $u_{0}(z)$ is a series in $z^{3}$ with $\mathfrak{v}\left(u_{0}(z)\right)=0$ (see [21, equation (8)] for the explicit expression of $u_{0}(z)$ ). Hence, it holds that

$$
U=\left\langle u_{0}(z), A u_{0}(z), \ldots, z^{2 n} u_{0}(z), z^{2 n} A u_{0}(z), \ldots\right\rangle .
$$

Therefore, as claimed and proved in [21], there is a unique $U$ stable by $A$ and $z^{-2}$ and it is explicitly given as above.

We now wish to rephrase these arguments in terms of Witt algebras. We wonder if there exists an action $(\mathbb{C}((z)), \rho)$ of $\mathcal{W}^{+}$leaving $U$ (the subspace of equation (4.5)) stable. The fact that $z^{-2} U \subset U$ implies that $h(z)=z^{-2}$. Since $U$ is $A$-stable, it follows that $A$ belongs to the first-order stabilizer of $U$ and, by the exact sequence (3.2) and since $\sigma(A)=-3^{\frac{2}{3}} \frac{1}{2} z^{3}$, there exists a polynomial $p(x)$ such that

$$
A=-3^{\frac{2}{3}} \rho\left(L_{-1}\right)+p(h(z)) .
$$

From where we obtain the equation

$$
-\frac{1}{2} z^{-1}+\frac{1}{4} z^{2}=-\frac{1}{2} z^{3} b(z)+p(h(z)) .
$$

We will consider the solution $b(z)=z^{-4}-\frac{1}{2} z^{-1}, p(h(z))=0$. Thus, we may assume that the action is defined by $h(z)=z^{-2}, c \in \mathbb{C}$ and $b(z)=z^{-4}-\frac{1}{2} z^{-1}$.

In [25], Kontsevich proved Witten's conjecture, which claims that a generating function for intersection indices coincides with the partition function in the standard matrix model theory. In particular, it has to obey Virasoro constraints and the KdV hierarchy. The action of these fields on the space of fermions corresponds to the following operators [7, equation (7.33)] (see also [40, equation (2.59)])

$$
\begin{aligned}
& \bar{L}_{-1}^{\prime}=-\frac{1}{2} \partial_{q_{0}}+\sum_{i=1}^{\infty}\left(i+\frac{1}{2}\right) q_{i} \partial_{q_{i-1}}+\frac{1}{4} q_{0}^{2}, \quad \bar{L}_{0}^{\prime}=-\frac{1}{2} \partial_{q_{1}}+\sum_{i=0}^{\infty}\left(i+\frac{1}{2}\right) q_{i} \partial_{q_{i}}+\frac{1}{16}, \\
& \bar{L}_{n}^{\prime}=-\frac{1}{2} \partial_{q_{n+1}}+\sum_{i=0}^{\infty}\left(i+\frac{1}{2}\right) q_{i} \partial_{q_{i+n}}+\frac{1}{4} \sum_{i=1}^{n} \partial_{q_{i-1}} \partial_{q_{n-i}}, \quad n \geq 1 .
\end{aligned}
$$

which have Lie bracket $\left[\bar{L}_{i}^{\prime}, \bar{L}_{j}^{\prime}\right]=(i-j) L_{i+j}$ for $i, j \geq-1$ and correspond to the following operators acting on $\mathbb{C}((z))$

$$
\tilde{L}_{n}:=\frac{1}{2} z^{-2 n}\left(z \partial_{z}+\frac{1-2 n}{2}\right)-\frac{1}{2} z^{-(2 n+3)}, \quad n \geq-1 .
$$


The Lie algebra generated by these operators is isomorphic to the Virasoro algebra and the Witten-Kontsevich $\tau$-function is annihilated by the operators $\left\{\tilde{L}_{n}\right\}$ and is uniquely characterized by this property up to a scalar factor (see [17, Section 3]).

In our setting

$$
-\frac{h(z)^{n+1}}{h^{\prime}(z)}=\frac{1}{2} z^{-2 n+1}, \quad-(n+1) \operatorname{ch}(z)^{n}+\frac{b(z)}{h^{\prime}(z)} h(z)^{n+1}=\frac{1}{2} z^{-(2 n+3)}-\frac{2 n-1}{4} z^{-2 n}
$$

and, therefore, $h(z)=z^{-2}, c=\frac{1}{2}$ and $b(z)=z^{-4}-\frac{3}{2} z^{-1}$.

Notice that in the case of Kac-Schwarz [21] we obtained $b(z)=z^{-4}-\frac{1}{2} z^{-1}$ while we now have $b(z)=z^{-4}-\frac{3}{2} z^{-1}$. Let us explain why this is not contradictory. The difference between both expressions is $z^{-1}$, such that if the first case corresponds to the action on a vector space $V$, the second one corresponds to the conjugated action by $z \in \mathbb{C}((z))$ (see Theorem 2.9). Bearing in mind how $\tau$-functions on different connected components are computed, it follows that the $\tau$-functions of both cases do coincide.

Remark 4.3. It is worth mentioning the paper [2]. Its Section 2 is concerned with three instances of functions that solve simultaneously the KP hierarchy as well as Virasoro constraints; namely, Witten-Kontsevich, Hurwitz and Hodge $\tau$-functions. For each case, he computes the so-called Kac-Schwarz operators; that is, those differential operators preserving the point of the Grassmannian defined by each of the above $\tau$-function. In the first case [2, Section 2.3], he obtains two Kac-Schwarz operators $a_{\mathrm{KW}}=\frac{1}{z}-z^{3} \frac{\partial}{\partial z}-\frac{z^{2}}{2}, b_{\mathrm{KW}}=z^{-2}$ which satisfy $\left[a_{\mathrm{KW}}, b_{\mathrm{KW}}\right]_{-}=2$. Now, it is clear that the subalgebra generated by them coincide with (3.1). Further $A_{U}^{1}$ is a subalgebra of the Heisenberg-Virasoro algebra (see equation (2.8)). It is quite relevant that in the other two cases, the operators $a_{\text {Hurwitz }}, b_{\text {Hurwitz }}$ and $a_{\text {Hodge }}, b_{\text {Hogde }}$ are conjugated with $a_{\mathrm{KW}}, b_{\mathrm{KW}}$.

\subsection{Spectral curves}

It is natural to wonder whether the subspace of Theorem 3.12 can be described in terms of algebraic geometry via the Krichever morphism.

We continue with an action of $\mathcal{W}^{+},(V=\mathbb{C}((z)), \rho)$, under the following assumptions: $h(z)=$ $z^{-2} ; v(z)$ and $u(x)$ satisfy equation (3.6) with $u^{\prime \prime}(x) \neq 0$; and the hypotheses of Theorem 3.12 hold. Let $U:=\mathcal{U}(w)$ be the point of $\operatorname{Gr}\left(V^{w v}\right)$ given by Theorem 3.12 for a solution $W$ of the Airy equation and let $A_{U}=\mathbb{C}\left[z^{-2}\right]$ be its stabilizer.

Recall that by applying Krichever theory to the pair $\left(A_{U}, U\right)$, one gets a rank 2 vector bundle on the projective line. Indeed, the projective line $\mathbb{P}_{1}$ is obtained by attaching to $\mathbb{A}_{1}=\operatorname{Spec} A_{U}$ the point defined by the valuation $\mathfrak{v}$. Let us set $\bar{z}:=h(z)$ as a coordinate in $\mathbb{P}_{1} \backslash\{\mathbf{0}\}=\mathbb{A}_{1}=$ Spec $A_{U}$. Moreover, the $A_{U}$-module $U$, being a point in $\operatorname{Gr}\left(V^{v}\right)$, gives rise to a rank 2 vector bundle on $\mathbb{P}_{1}, \mathcal{E}$. Since $U$ is generated by $1 \otimes v(z)$ and $\rho^{v}\left(L_{-1}\right)(1 \otimes v(z))$ as a module over $A_{U}=H^{0}\left(\mathbb{P}_{1} \backslash\{\mathbf{0}\}, \mathcal{O}_{\mathbb{P}_{1}}\right)$, let us introduce $T_{\rho}$, an endomorphism of $U$ as an $A_{U}$-module, by

$$
\begin{aligned}
& T_{\rho}(1 \otimes v(z)):=\rho^{v}\left(L_{-1}\right)(1 \otimes v(z)), \\
& T_{\rho}\left(\rho^{v}\left(L_{-1}\right)(1 \otimes v(z))\right):=\rho^{v}\left(L_{-1}\right)^{2}(1 \otimes v(z)) .
\end{aligned}
$$

In this case, $T_{\rho}$ is homogeneous of degree $d$ w.r.t. the filtration $U \cap \bar{z}^{i} \mathbb{C}[[z]]$. Thus, it yields a map

$$
\mathcal{E} \longrightarrow \mathcal{E} \otimes \mathcal{O}_{\mathbb{P}_{1}}(d)
$$

of $\mathcal{O}_{\mathbb{P}_{1}}$-modules or, equivalently, a map

$$
\operatorname{Sym} \mathcal{O}_{\mathbb{P}_{1}}(-d) \longrightarrow \operatorname{End}(\mathcal{E})
$$

of sheaves of $\mathcal{O}_{\mathbb{P}_{1}}$-algebras. 
Recalling the construction of the spectral curve [4], these data define a 2:1-covering $\pi_{\rho}: X_{\rho} \rightarrow$ $\mathbb{P}_{1}$ and a rank 1 torsion free sheaf $\mathcal{L}_{\rho}$ on $X_{\rho}$ such that $\left(\pi_{\rho}\right)_{*} \mathcal{L}_{\rho}=\mathcal{E}$.

Let us look closer at this spectral curve. Recalling the explicit computations of the proof of Lemma 3.10, it holds that $T_{\rho}\left(\rho^{v}\left(L_{-1}\right)(1 \otimes v(z))\right)=-\left(u(\bar{z})^{2}+u^{\prime}(\bar{z})\right) \otimes v(z)+2 u(\bar{z}) \rho^{v}\left(L_{-1}\right)(1 \otimes$

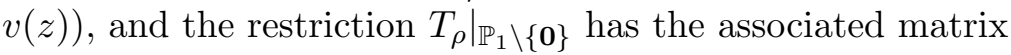

$$
\left(\begin{array}{cc}
0 & -u(\bar{z})^{2}-u^{\prime}(\bar{z}) \\
1 & 2 u(\bar{z})
\end{array}\right)
$$

w.r.t. the basis $\left\{1 \otimes v(z), \rho^{v}\left(L_{-1}\right)(1 \otimes v(z))\right\}$ and thus the characteristic polynomial of $T_{\rho}$ is

$$
\operatorname{char}_{T_{\rho}}(y)=y^{2}-2 u(\bar{z}) y+\left(u(\bar{z})^{2}+u^{\prime}(\bar{z})\right) .
$$

Hence, $X_{\rho}$ is an integral curve as long as $u^{\prime}(x)$ is not a square. Furthermore, observe that

$$
X_{\rho}-\pi_{\rho}^{-1}(\{\mathbf{0}\}) \simeq \operatorname{Spec} \mathbb{C}[X, Y] /\left(Y^{2}-2 u(X) Y+\left(u(X)^{2}+u^{\prime}(X)\right)\right),
$$

where the ring of the r.h.s. can be thought as a subalgebra of $\operatorname{End}_{A_{U}}(U)$ identifying $X$ with the homothety of ratio $\bar{z}=h(z)$ and $Y$ with the endomorphism $T_{\rho}$. Finally, the branch locus of $\pi_{\rho}$ consists of the zero locus of $u^{\prime}(\bar{z})$ (recall that $\bar{z}$ is a coordinate in $\mathbb{P}_{1} \backslash\{\mathbf{0}\}=\operatorname{Spec} A_{U}$ ) and the point $\mathbf{0}$. The singular points of $X_{\rho}$ are precisely the preimages of the locus of multiple roots of $u^{\prime}(\bar{z})=0$ together with the point $\pi_{\rho}^{-1}(\{\mathbf{0}\})$ (if $d>1$ ). Hence, $X_{\rho}$ is a hyperelliptic curve and its genus can be easily expressed in terms of $u(\bar{z})$.

The Hitchin map provided by the theory of spectral curves takes the form

$$
\rho \rightsquigarrow\left(2 u(\bar{z}),-u(\bar{z})^{2}-u^{\prime}(\bar{z})\right),
$$

whose fibers are essentially given by Jacobian varieties. Let us see this fact by studying $b(z)$. Indeed, if we express $b(z)$ as in equation (3.6)

$$
b(z)=u(h(z)) h^{\prime}(z)+\left(\frac{v^{\prime}(z)}{v(z)}-\frac{1}{2} h^{\prime \prime}(z)\right)
$$

w.r.t. the splitting $\mathbb{C}((z))=\mathbb{C}[h(z)] h^{\prime}(z) \oplus\left(\mathbb{C}[h(z)]+z^{-1} \mathbb{C}[[z]]\right)$ we observe that varying the coefficients of $u$ may vary the spectral curve but not the $\tau$-function (see Theorem 3.17 ) while the variation of the second term, $\frac{v^{\prime}(z)}{v(z)}-\frac{1}{2} h^{\prime \prime}(z)$, leaves $X_{\rho}$ fixed but may change the sheaf $\mathcal{L}_{\rho}$ (this will be discussed in Section 4.4). In other words, the variation of $u(\bar{z})$ would correspond to monodromy-preserving tau functions and, hence, would be related to Painlevé equations while the variation of the second term are KP flows.

Remark 4.4. Note that most of the examples given in [12, Section 10] are based on curves that have the form of equation (4.7) and that, in certain cases [11, Sections 2 and 3], the desired $\tau$-function, satisfying Virasoro constraints and KdV hierarchy, can be expressed in terms of the theta function of a hyperelliptic curve (namely, $X_{\rho}$ ). Thus, we shall investigate in a future paper the relation between the spectral curves and $\tau$-functions of our approach and the spectral curves and $\tau$-functions of Eynard-Orantin $[12,13]$ since we expect that both constructions are deeply connected.

Remark 4.5. Very recently Schwarz has published [38]. In his Section 2 he shows that for a point $U$ in the Grassmannian invariant under $z^{q}$ and $\hbar \frac{d}{d z^{p}}+b(z)$ there are two differential operators $P, Q$ s.t. $[P, Q]=\hbar$ and that they determine a quantum curve. Observe that the study of this situation is almost equivalent, thanks to Proposition 3.15, to the study of the case $\hbar=1$ (see [37]). Further, he considers a $z^{q}$-basis of $U$; that is, vectors $v_{0}, \ldots, v_{q-1}$ such that $\left\{z^{m q} v_{n}\right\}$ generate $U$. It is easy to check that, for $q=2$, his $U$ coincide with our $U$ above and his equation (3) with our (4.6). 


\subsection{Opers on the punctured disk}

Regarding the definition and properties of the opers, which were introduced by Drinfeld and Sokolov and generalized by Beilinson and Drinfeld, we refer interested readers to [14, 15]. For our own case, it suffices to recall that a $\mathrm{Gl}(n)$-oper on the punctured disk, $D^{\times}$, is a rank $n$ vector bundle $\mathcal{E}$ on $D^{\times}$equipped with a flag $0 \subset \mathcal{E}_{1} \subset \cdots \subset \mathcal{E}_{n-1} \subset \mathcal{E}_{n}=\mathcal{E}$ of subbundles and a flat connection $\nabla: \mathcal{E} \rightarrow \mathcal{E} \otimes \Omega_{D^{\times}}$such that $\nabla\left(\mathcal{E}_{i}\right) \subseteq \mathcal{E}_{i+1} \otimes \Omega_{D^{\times}}$and the induced maps $\mathcal{E}_{i} / \mathcal{E}_{i-1} \rightarrow\left(\mathcal{E}_{i+1} / \mathcal{E}_{i}\right) \otimes \Omega_{D^{\times}}$are a isomorphisms of $\mathcal{O}_{D^{\times}}$-bundles for all $i$ (transversality).

Let us see how we can associate an oper on the punctured disc $D^{\times}$with certain actions and conversely (see [36] for a detailed exposition).

Let $(V=\mathbb{C}((z)), \rho)$ be an action of $\mathcal{W}^{+}$such that $h(z)=z^{-2}$. Let $U:=\mathcal{U}(w)$ be the point of $\operatorname{Gr}\left(V^{w v}\right)$ given by Theorem 3.12 and let $A_{U}=\mathbb{C}\left[z^{-2}\right]$ be its stabilizer.

First, let $\mathcal{E}$ be the vector bundle on $\operatorname{Spec} \mathbb{C}\left(\left(h(z)^{-1}\right)\right)$ defined by $\mathbb{C}((z))$. Hence, the subbundles $\mathcal{E}_{i}$, associated with

$$
\mathbb{C}\left(\left(h(z)^{-1}\right)\right) \otimes_{\mathbb{C}}\left\langle 1, \rho\left(L_{-1}\right)(1), \ldots, \rho\left(L_{-1}\right)^{i-1}(1)\right\rangle \subseteq \mathbb{C}((z)), \quad i=1,2
$$

define a flag of vector bundles $0 \subset \mathcal{E}_{1} \subset \mathcal{E}_{2}=\mathcal{E}$.

Let us see that $\mathcal{E}$ carries a connection. Indeed, let $\mathrm{d}: \mathbb{C}\left(\left(h(z)^{-1}\right)\right) \rightarrow \Omega_{\mathbb{C}\left(\left(h(z)^{-1}\right)\right) / \mathbb{C}}$ be the differential and consider the $\mathbb{C}$-linear map $\rho\left(L_{-1}\right) \otimes \mathrm{d} h-1 \otimes \mathrm{d}$ :

$$
\begin{aligned}
& \mathbb{C}((z)) \otimes_{\mathbb{C}} \mathbb{C}\left(\left(h(z)^{-1}\right)\right) \longrightarrow \mathbb{C}((z)) \otimes_{\mathbb{C}} \Omega_{\mathbb{C}\left(\left(h(z)^{-1}\right)\right) / \mathbb{C}}, \\
& f \otimes a \longmapsto \rho\left(L_{-1}\right) f \otimes a \mathrm{~d} h-f \otimes \mathrm{d} a .
\end{aligned}
$$

One checks that when composing with the canonical map

$$
\mathbb{C}((z)) \otimes_{\mathbb{C}} \Omega_{\mathbb{C}\left(\left(h(z)^{-1}\right)\right) / \mathbb{C}} \rightarrow \mathbb{C}((z)) \otimes_{\mathbb{C}\left(\left(h(z)^{-1}\right)\right)} \Omega_{\mathbb{C}\left(\left(h(z)^{-1}\right)\right) / \mathbb{C}}
$$

the images of $f \otimes a$ and of $a f \otimes 1$ do coincide and, therefore, we obtain a connection $\nabla: \mathcal{E} \rightarrow$ $\mathcal{E} \otimes \Omega_{\operatorname{Spec}} \mathbb{C}\left(\left(h(z)^{-1}\right)\right)$ that satisfies the transversality condition.

Let us see a converse; that is, given a $\mathrm{Gl}(2)$-oper on $D^{\times}$one can define an action of $\mathcal{W}^{+}$. Let $(\mathcal{E}, \nabla)$ the $\operatorname{Gl}(2)$-oper on $D^{\times}=\operatorname{Spec}\left(\mathbb{C}\left(\left(h(z)^{-1}\right)\right)\right)$ where $h(z):=z^{-2}$. Let $\langle$,$\rangle be the pairing of$ differentials with derivations. It can be shown that

$$
\nabla_{D}(f):=\langle\nabla f, D\rangle \quad \text { for } f \in \mathbb{C}((z)), \quad D \in \operatorname{Der}\left(\mathbb{C}\left(\left(h(z)^{-1}\right)\right)\right)
$$

is a differential operator of $\mathbb{C}((z))$ as a $\mathbb{C}\left(\left(h(z)^{-1}\right)\right)$-module. For $D_{i}:=-\frac{h(z)^{i+1}}{h^{\prime}(z)} \frac{\partial}{\partial z}$, let us consider the linear map

$$
\begin{aligned}
& \mathcal{W}^{+} \stackrel{\rho}{\longrightarrow} \mathcal{D}_{\mathbb{C}((z)) / \mathbb{C}}^{1}(\mathbb{C}((z)), \mathbb{C}((z))), \\
& L_{i} \mapsto \rho\left(L_{i}\right):=\nabla_{D_{i}} .
\end{aligned}
$$

The fact that $\rho$ is a morphism of Lie algebras is derived from the flatness of $\nabla$ as follows

$$
\left[\rho\left(L_{i}\right), \rho\left(L_{j}\right)\right]=\left[\nabla_{D_{i}}, \nabla_{D_{j}}\right]=\nabla_{\left[D_{i}, D_{j}\right]}=\nabla_{(i-j) D_{i+j}}=(i-j) \rho\left(L_{i+j}\right) .
$$

However, the constructed oper is defined up to certain gauge transformations; namely, conjugation by Aut $\mathbb{C}_{\text {-alg }} \mathbb{C}((\bar{z}))$ (in order to identify $\mathbb{C}((\bar{z}))$ and $\mathbb{C}\left(\left(h(z)^{-1}\right)\right)$ ) and by homotheties by $\mathbb{C}((z))$ (in order to identify $V$ and $\mathbb{C}((z)))$. The effects of these transformations have been studied in Section 2.2.

Remark 4.6. Observe that, in the case $A_{U}=\mathbb{C}[h(z)]$, the connection can be introduced in an alternative way. Indeed, the map $h(z)^{n} \partial_{h} \mapsto L_{n-1}$, for $n \geq 0$, provides a section of the canonical $\operatorname{map} \mathcal{D}_{A_{U} / \mathbb{C}}^{1}(U) \rightarrow \operatorname{Der}_{\mathbb{C}}\left(A_{U}\right)$ which, by [5, Section 1.1]), is an integrable connection on $\mathcal{E}$ on $\operatorname{Spec} \mathbb{C}\left(\left(h(z)^{-1}\right)\right)$. 
Remark 4.7. The techniques of [15, Chapter 5] can be applied to the above results in order to associate to a a general action $(V, \rho)$ a $\mathrm{Gl}(n)$-oper on the abstract punctured disk $D^{\times}=$ Spec $\mathbb{C}((\bar{z}))$ (or, even, on an algebraic curve). On the other hand, recalling from $[19,20]$ the close relationship between vertex algebras and infinite-dimensional representations of the Virasoro algebra, we expect to interpret the action of $\mathcal{W}^{+}$on $\mathbb{C}\left[\left[t_{1}, t_{2}, \ldots\right]\right]$ in terms of vertex operators. This will help to understand our approach within Frenkel's framework of the geometric Langlands program [14].

\subsection{Universal family}

Recent results on intersection theory are based on the so-called topological recursion [23, 27, $29,30,32,33]$ which are formulae involving families of $\tau$-functions depending on an infinite number of parameters such that the whole family lies entirely on the space of functions satisfying $\mathrm{KdV}$ and Virasoro constraints. One of these families already appeared in Kontsevich's work [25, Section 3.4]. It is worth mentioning the existence of relevant 1-parameter families; for instance, the one connecting Witten-Kontsevich and Mirzakhani theories [32], and another one the Witten-Kontsevich partition function with the generating function of linear Hodge integrals defined on the moduli space of stable curves [23].

It would be very interesting to generalize our methods to include the whole KP hierarchy and to interpret the family connecting the Witten-Kontsevich partition function with the Hurwitz partition function [28] (see also [6]). A recent result on this direction is in [2] to be found.

In this subsection, a natural procedure to obtain the above mentioned families will be provided. Indeed, the proposed family will consist of a more general expression for $b(z)$ under the constraint that $b_{-1} \in \frac{1}{2} \mathbb{Z} \backslash \mathbb{Z}$.

Let us consider a family of independent variables $\mathbf{s}:=\left(s_{1}, s_{2}, \ldots\right)$. For a sequence of nonnegative integers, $\mathbf{m}:=\left(m_{1}, m_{2}, \ldots\right)$, with $m_{i}=0$ for all $i \gg 0$ define

$$
|\mathbf{m}|:=\sum_{i \geq 1} i m_{i}, \quad\|\mathbf{m}\|:=\sum_{i \geq 1} m_{i}, \quad \mathbf{m} !:=\prod_{i \geq 1} m_{i} !, \quad \mathbf{s}^{\mathbf{m}}:=\prod_{i \geq 1} s_{i}^{m_{i}} .
$$

Based on Mulase-Safnuk's approach [32], Liu-Xu considered the operators [27, equation (9)]

$$
\begin{aligned}
\bar{L}_{n}^{\prime}(\mathbf{s}):= & -\frac{1}{2} \sum_{\mathbf{m}} \frac{(-1)^{\|\mathbf{m}\|}}{\mathbf{m} !(2|\mathbf{m}|+1) ! !} \mathbf{s}^{\mathbf{m}} \partial_{q_{|\mathbf{m}|+n+1}}+\sum_{i=0}^{\infty}\left(i+\frac{1}{2}\right) q_{i} \partial_{q_{i+n}} \\
& +\frac{1}{2} \sum_{i=1}^{n} \partial_{q_{i-1}} \partial_{q_{n-i}}+\frac{q_{0}^{2}}{4} \delta_{n,-1}+\frac{1}{16} \delta_{n, 0}
\end{aligned}
$$

for $n \geq-1$ (their exact expression corresponds to a rescaling by a double factorial). They showed that

$$
\left[\bar{L}_{i}^{\prime}(\mathbf{s}), \bar{L}_{j}^{\prime}(\mathbf{s})\right]=(i-j) \bar{L}_{i+j}^{\prime}(\mathbf{s}) \quad \text { for } \quad i, j \geq-1
$$

and, therefore, they generate a family of Witt algebras depending on the parameters $\mathbf{s}$.

Observe that the operators $\bar{L}_{n}^{\prime}(0)$, i.e., $\bar{L}_{n}^{\prime}(\mathbf{s})$ for $\mathbf{s}=0$, coincide with those of equation (4.3) for $\lambda=2^{-\frac{1}{2}}$ (up to rescaling of the variables $q_{i}$ ). That is, we have

$$
\bar{L}_{n}^{\prime}(\mathbf{s})=-\frac{1}{2} \sum_{\mathbf{m}} \frac{(-1)^{\|\mathbf{m}\|}}{\mathbf{m} !(2|\mathbf{m}|+1) ! !} \mathbf{s}^{\mathbf{m}} \partial_{q_{|\mathbf{m}|+n+1}}+\bar{L}_{n}^{\prime}(0) .
$$

Bearing in mind that the action induced by $\bar{L}_{n}^{\prime}(0)$ on $\mathbb{C}((z))$ can be obtained by arguments analogous to those of the equation (4.4), we obtain the action corresponding to the above 
operators by replacing $q_{m}$ by $t_{2 m+1}$ and $\partial_{t_{2 m+1}}$ by $z^{-2 m-1}$ :

$$
\rho_{\mathbf{s}}^{\prime}\left(L_{n}\right):=\frac{1}{2} \sum_{\mathbf{m}} \frac{(-1)^{\|\mathbf{m}\|}}{\mathbf{m} !(2|\mathbf{m}|+1) ! !} \mathbf{s}^{\mathbf{m}} z^{-2(|\mathbf{m}|+n)-3}+\frac{1}{2} z^{-2 n}\left(z \partial_{z}+\frac{1-2 n}{2}\right) \quad \forall n \geq-1 .
$$

Since $\frac{h(z)^{n+1}}{h^{\prime}(z)}=-\frac{1}{2} z^{-2 n+1}$, and regarding $\mathbf{s}$ as parameters, we obtain that the action $\rho_{\mathbf{s}}^{\prime}$ is attached to the data $\left(h(z)=z^{-2}, c=\frac{1}{2}, b_{\mathbf{s}}(z)\right)$, where

$$
b_{\mathbf{s}}(z):=-\sum_{\mathbf{m}} \frac{(-1)^{\|\mathbf{m}\|}}{\mathbf{m} !(2|\mathbf{m}|+1) ! !} \mathbf{s}^{\mathbf{m}} z^{-2|\mathbf{m}|-4}-\frac{3}{2} z^{-1} .
$$

It must be pointed out that, bearing in mind Theorem 2.9, Proposition 3.6 and Theorem 3.17, we conclude that the action $\bar{\rho}_{\mathbf{S}}^{\prime}$ (i.e., that induced by $\rho_{\mathbf{S}}^{\prime}$ on $\mathbb{C}\left[\left[t_{1}, t_{3}, \ldots\right]\right]$ ) is the universal action for the case of $\mathrm{KdV}$ (i.e., $h(z)=z^{-2}$ ). In particular, this agrees with the idea addressed in [32] that a certain 1-parameter family, which would correspond to Eynard's spectral curve, deforms the Witten-Kontsevich theory to other cases where the Virasoro also appears. Thus, our techniques provide an alternative proof of [32, Theorem 4.4] (see also [23, Theorem 2.1] and [27, Theorem 4.1]).

Theorem 4.8. Let $\tau_{\mathbf{s}}(t) \in \mathbb{C}\left[\left[t_{1}, t_{3}, \ldots\right]\right]$ be the $\tau$-function associated to $\rho_{\mathbf{s}}^{\prime}$. Then, $\tau_{\mathbf{s}}(t)$ satisfies the Virasoro constraints corresponding to operators $\bar{L}_{n}^{\prime}(\mathbf{s})$ above (as in Section 3.3) and, moreover, it holds that

$$
\tau_{\mathbf{s}}(t)=\tau_{0}(\tilde{t}),
$$

where $\tilde{t}_{2+1}$ is equal to $t_{i}$ for $i=0,1$ and to $t_{2 i+1}-\frac{1}{(2 i+1) ! !} \sum_{|\mathbf{m}|=i-1} \frac{(-1)^{\|\mathbf{m}\|}}{\mathbf{m} !} \mathbf{s}^{\mathbf{m}}$ for $i>1$.

Conversely, let $\tau(t) \in \mathbb{C}\left[\left[t_{1}, t_{3}, \ldots\right]\right]$ be a $\tau$-function for the $K d V$ satisfying the Virasoro constraints. Then, there exist values of $\mathbf{s}$, say $\mathbf{s}_{0}:=\left(s_{1}, s_{2}, \ldots\right)$ such that

$$
\tau(t)=\tau_{\mathbf{s}_{0}}(t) .
$$

Proof. It is enough to observe that under that change of variables, the operators $\bar{L}_{n}^{\prime}(\mathbf{s})$ in $t_{2 i+1}$ are transformed into the operators $\bar{L}_{n}^{\prime}(0)$ in $\tilde{t}_{2 i+1}$. Bearing in mind that, regarding $\mathbf{s}$ as parameters, the action $\rho_{\mathbf{s}}^{\prime}$ is universal, the converse follows.

Finally, note that the variation of $\mathbf{s}$ is equivalent to the variation of the line bundle constructed in Section 4.2. Further, the previous approach can be also understood by replacing the base field $\mathbb{C}$ by the ring $\mathbb{C}\left[\left[s_{1}, s_{2}, \ldots\right]\right]$. Then, the resulting framework would make use of the relative Sato Grassmannian while the change of variables $t_{2+1}$ by $\tilde{t}_{2+1}$ corresponds to a trivialization of the relative Grassmannian [35].

\section{Acknowledgements}

I wish to thank Edward Frenkel, Esteban Gómez and, very specially, Motohico Mulase for stimulating discussions and to the referees for their remarks.

\section{References}

[1] Adler M., Morozov A., Shiota T., van Moerbeke P., A matrix integral solution to $[P, Q]=P$ and matrix Laplace transforms, Comm. Math. Phys. 180 (1996), 233-263, hep-th/9610137.

[2] Alexandrov A., Enumerative geometry, tau-functions and Heisenberg-Virasoro algebra, Comm. Math. Phys. 338 (2015), 195-249, arXiv:1404.3402. 
[3] Álvarez Vázquez A., Muñoz Porras J.M., Plaza Martín F.J., The algebraic formalism of soliton equation over arbitrary base fields, in Taller de Variedades Abelianas y Funciones Theta (Morelia, 1996), Aportaciones Matemáticas: Investigación, Vol. 13, Editors R. Rodríguez, J.M. Muñóz Porras, S. Recillas, Sociedad Matemática Mexicana, México, 1998, 3-40, alg-geom/9606009.

[4] Beauville A., Narasimhan M.S., Ramanan S., Spectral curves and the generalised theta divisor, J. Reine Angew. Math. 398 (1989), 169-179.

[5] Beŭlinson A.A., Schechtman V.V., Determinant bundles and Virasoro algebras, Comm. Math. Phys. 118 (1988), 651-701.

[6] Bouchard V., Mariño M., Hurwitz numbers, matrix models and enumerative geometry, in From Hodge Theory to Integrability and TQFT $t t^{*}$-Geometry, Proc. Sympos. Pure Math., Vol. 78, Amer. Math. Soc., Providence, RI, 2008, 263-283, arXiv:0709.1458.

[7] Dijkgraaf R., Intersection theory, integrable hierarchies and topological field theory, in New Symmetry Principles in Quantum Field Theory (Cargèse, 1991), NATO Adv. Sci. Inst. Ser. B Phys., Vol. 295, Plenum, New York, 1992, 95-158, hep-th/9201003.

[8] Dijkgraaf R., Verlinde H., Verlinde E., Loop equations and Virasoro constraints in nonperturbative twodimensional quantum gravity, Nuclear Phys. B 348 (1991), 435-456.

[9] Douglas M.R., Strings in less than one dimension and the generalized KdV hierarchies, Phys. Lett. B 238 (1990), 176-180.

[10] Dubrovin B., Zhang Y., Frobenius manifolds and Virasoro constraints, Selecta Math. (N.S.) 5 (1999), 423466, math.AG/9808048.

[11] Eynard B., Mariño M., A holomorphic and background independent partition function for matrix models and topological strings, J. Geom. Phys. 61 (2011), 1181-1202, arXiv:0810.4273.

[12] Eynard B., Orantin N., Invariants of algebraic curves and topological expansion, Commun. Number Theory Phys. 1 (2007), 347-452, math-ph/0702045.

[13] Eynard B., Orantin N., Geometrical interpretation of the topological recursion, and integrable string theories, arXiv:0911.5096.

[14] Frenkel E., Langlands correspondence for loop groups, Cambridge Studies in Advanced Mathematics, Vol. 103, Cambridge University Press, Cambridge, 2007.

[15] Frenkel E., Ben-Zvi D., Vertex algebras and algebraic curves, Mathematical Surveys and Monographs, Vol. 88, 2nd ed., Amer. Math. Soc., Providence, RI, 2004.

[16] Frenkel E., Kac V., Radul A., Wang W., $\mathcal{W}_{1+\infty}$ and $\mathcal{W}\left(\mathfrak{g l}_{N}\right)$ with central charge N, Comm. Math. Phys. 170 (1995), 337-357, hep-th/9405121.

[17] Givental A.B., Gromov-Witten invariants and quantization of quadratic Hamiltonians, Mosc. Math. J. 1 (2001), 551-568, math.AG/0108100.

[18] Gómez González E., Hernández Serrano D., Muñoz Porras J.M., Plaza Martín F.J., Geometric approach to Kac-Moody and Virasoro algebras, J. Geom. Phys. 62 (2012), 1984-1997.

[19] Kac V.G., Peterson D.H., Spin and wedge representations of infinite-dimensional Lie algebras and groups, Proc. Nat. Acad. Sci. USA 78 (1981), 3308-3312.

[20] Kac V.G., Raina A.K., Bombay lectures on highest weight representations of infinite-dimensional Lie algebras, Advanced Series in Mathematical Physics, Vol. 2, World Scientific Publishing Co., Inc., Teaneck, NJ, 1987.

[21] Kac V.G., Schwarz A., Geometric interpretation of the partition function of 2D gravity, Phys. Lett. B 257 (1991), 329-334.

[22] Katsura T., Shimizu Y., Ueno K., Complex cobordism ring and conformal field theory over Z, Math. Ann. 291 (1991), 551-571.

[23] Kazarian M., KP hierarchy for Hodge integrals, Adv. Math. 221 (2009), 1-21, arXiv:0809.3263.

[24] Kirillov A.A., Geometric approach to discrete series of unirreps for Vir, J. Math. Pures Appl. 77 (1998), 735-746.

[25] Kontsevich M., Intersection theory on the moduli space of curves and the matrix Airy function, Comm. Math. Phys. 147 (1992), 1-23.

[26] Laine I., Nevanlinna theory and complex differential equations, de Gruyter Studies in Mathematics, Vol. 15, Walter de Gruyter \& Co., Berlin, 1993. 
[27] Liu K., Xu H., Recursion formulae of higher Weil-Petersson volumes, Int. Math. Res. Not. 2009 (2009), 835-859, arXiv:0708.0565.

[28] Mironov A., Morozov A., Virasoro constraints for Kontsevich-Hurwitz partition function, J. High Energy Phys. 2009 (2009), no. 2, 024, 52 pages, arXiv:0807.2843.

[29] Mirzakhani M., Simple geodesics and Weil-Petersson volumes of moduli spaces of bordered Riemann surfaces, Invent. Math. 167 (2007), 179-222.

[30] Mirzakhani M., Weil-Petersson volumes and intersection theory on the moduli space of curves, J. Amer. Math. Soc. 20 (2007), 1-23.

[31] Mulase M., Algebraic theory of the KP equations, in Perspectives in mathematical physics, Conf. Proc. Lecture Notes Math. Phys., III, Int. Press, Cambridge, MA, 1994, 151-217.

[32] Mulase M., Safnuk B., Mirzakhani's recursion relations, Virasoro constraints and the KdV hierarchy, Indian J. Math. 50 (2008), 189-218, math.QA/0601194.

[33] Mulase M., Zhang N., Polynomial recursion formula for linear Hodge integrals, Commun. Number Theory Phys. 4 (2010), 267-293, arXiv:0908.2267.

[34] Muñoz Porras J.M., Plaza Martín F.J., Automorphism group of $k((t))$ : applications to the bosonic string, Comm. Math. Phys. 216 (2001), 609-634, hep-th/9903250.

[35] Plaza Martín F.J., Arithmetic infinite Grassmannians and the induced central extensions, Collect. Math. 61 (2010), 107-129, arXiv:0810.0354.

[36] Plaza Martín F.J., Representations of the Witt algebra and Gl(n)-opers, Lett. Math. Phys. 103 (2013), 1079-1101.

[37] Schwarz A., On solutions to the string equation, Modern Phys. Lett. A 6 (1991), 2713-2725, hep-th/9109015.

[38] Schwarz A., Quantum curves, Comm. Math. Phys. 338 (2015), 483-500, arXiv:1401.1574.

[39] Segal G., Wilson G., Loop groups and equations of KdV type, Inst. Hautes Études Sci. Publ. Math. (1985), $5-65$.

[40] Witten E., Two-dimensional gravity and intersection theory on moduli space, in Surveys in Differential Geometry (Cambridge, MA, 1990), Lehigh Univ., Bethlehem, PA, 1991, 243-310. 